\title{
THE
}

\section{From atomistic lattice-gas models for surface reactions to hydrodynamic reaction-diffusion equations}

\author{
J. W. Evans \\ Da-Jiang Liu \\ M. Tammaro \\ University of Rhode Island, tammaro@uri.edu
}

Follow this and additional works at: https://digitalcommons.uri.edu/phys_facpubs

Terms of Use

All rights reserved under copyright.

\section{Citation/Publisher Attribution}

Evans, J. W., Liu, D-J., \& Tammaro, M. (2002). From atomistic lattice-gas models for surface reactions to hydrodynamic reaction-diffusion equations. Chaos: An Interdisciplinary Journal of Nonlinear Science, 12(1), 131-143. doi: 10.1063/1.1450566

Available at: https://doi.org/10.1063/1.1450566

This Article is brought to you for free and open access by the Physics at DigitalCommons@URI. It has been accepted for inclusion in Physics Faculty Publications by an authorized administrator of DigitalCommons@URI. For more information, please contact digitalcommons-group@uri.edu. 


\section{From atomistic lattice-gas models for surface reactions to hydrodynamic reaction- diffusion equations}

J. W. Evans, Da-Jiang Liu, and M. Tammaro

Citation: Chaos 12, 131 (2002); doi: 10.1063/1.1450566

View online: https://doi.org/10.1063/1.1450566

View Table of Contents: http://aip.scitation.org/toc/cha/12/1

Published by the American Institute of Physics

\section{Articles you may be interested in}

Parallel kinetic Monte Carlo simulation framework incorporating accurate models of adsorbate lateral interactions The Journal of Chemical Physics 139, 224706 (2013); 10.1063/1.4840395

Stochastic simulation of catalytic surface reactions in the fast diffusion limit The Journal of Chemical Physics 125, 194715 (2006); 10.1063/1.2390696

Kinetic phase diagrams for the monomer-dimer surface reaction: Unification of mean-field and lattice-gas behavior

The Journal of Chemical Physics 97, 572 (1992); 10.1063/1.463552

CO-oxidation model with superlattice ordering of adsorbed oxygen. I. Steady-state bifurcations The Journal of Chemical Physics 111, 6579 (1999); 10.1063/1.479949

Assessment of mean-field microkinetic models for CO methanation on stepped metal surfaces using accelerated kinetic Monte Carlo

The Journal of Chemical Physics 147, 152705 (2017); 10.1063/1.4989511

Beyond mean-field approximations for accurate and computationally efficient models of on-lattice chemical kinetics

The Journal of Chemical Physics 147, 024105 (2017); 10.1063/1.4991690

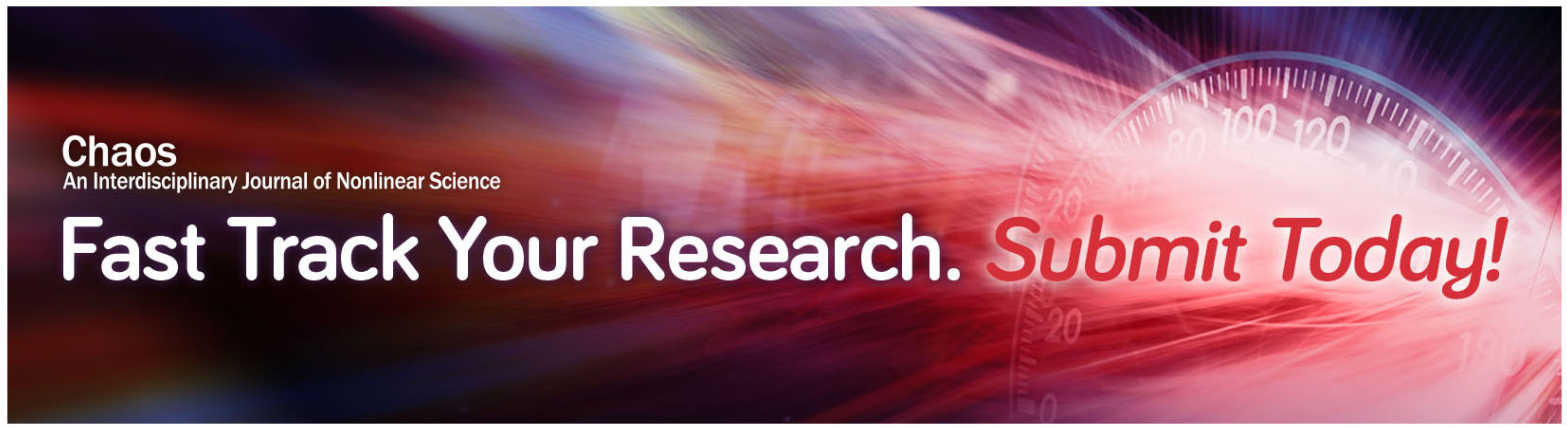




\title{
From atomistic lattice-gas models for surface reactions to hydrodynamic reaction-diffusion equations
}

\author{
J. W. Evans ${ }^{a)}$ \\ Ames Laboratory (USDOE) and Department of Mathematics, Iowa State University, Ames, Iowa 50011 \\ Da-Jiang Liu \\ Ames Laboratory (USDOE), Iowa State University, Ames, Iowa 50011 \\ M. Tammaro \\ Department of Physics, University of Rhode Island, Kingston, Rhode Island 02881
}

(Received 18 July 2001; accepted 20 December 2001; published 21 February 2002)

\begin{abstract}
Atomistic lattice-gas models for surface reactions can accurately describe spatial correlations and ordering in chemisorbed layers due to adspecies interactions or due to limited mobility of some adspecies. The primary challenge in such modeling is to describe spatiotemporal behavior in the physically relevant "hydrodynamic" regime of rapid diffusion of (at least some) reactant adspecies. For such models, we discuss the development of exact reaction-diffusion equations (RDEs) describing mesoscale spatial pattern formation in surface reactions. Formulation and implementation of these RDEs requires detailed analysis of chemical diffusion in mixed reactant adlayers, as well as development of novel hybrid and parallel simulation techniques. (C) 2002 American Institute of Physics. [DOI: 10.1063/1.1450566]
\end{abstract}

Local adsorption, desorption, and reaction processes occurring in surface reactions, when combined with surface diffusion, produce a diverse variety of spatiotemporal pattern formation. A key feature of these systems is that both the hysteresis often observed in the reaction kinetics, and the characteristic length of spatial patterns on the order of $\mu \mathrm{m}$, are controlled by the very rapid surface diffusion of at least some reactant adspecies. Many aspects of these phenomena have been successfully elucidated via mean-field reaction-diffusion equation modeling, in which the effects of adlayer ordering are neglected or treated approximately. A more fundamental approach is presented here based on atomistic lattice-gas (LG) models. One could attempt direct simulation of atomistic LG models, but this approach is complicated by the large separation of time and length scales (due to adspecies hop rates many orders of magnitude above other rates). Thus, we have developed another more appropriate strategy to "exactly" connect-the-length-scales from these atomistic LG models to the mesoscale pattern formation. Specifically, we treat directly this "hydrodynamic" regime of large hop rates utilizing special simulation models and procedures, coupled with a correct description of chemical diffusion in mixed reactant adlayers. This leads to development of exact reaction-diffusion equations for the hydrodynamic regime. In this report, we pay particular attention to recent developments in the description of chemical diffusion, which has a complicated tensorial nature since the presence of one adspecies can interfere with the diffusion of coadsorbed adspecies.

${ }^{\text {a)} E l e c t r o n i c ~ m a i l: ~ e v a n s @ a m e s l a b . g o v ~}$

\section{INTRODUCTION}

Many studies of surface reactions throughout the 1980s focused on the rich nonlinear kinetics observed under ultrahigh vacuum conditions on single crystal substrates. ${ }^{1}$ Substantial insight into this behavior has been derived from modeling using appropriate mean-field (MF) rate equations together with concepts from nonlinear dynamics to describe chemical kinetics. More recent application, primarily in the 1990s, of photoemission electron microscopy and lowenergy electron microscopy to surface reactions has revealed a diverse variety of spatiotemporal pattern formation and chemical wave propagation, prompting extensive and effective application of MF reaction-diffusion equation (RDE) modeling. ${ }^{2}$ A classic example illustrating these various concepts is bistability in CO-oxidation on $\mathrm{Pt}(111)$ for sufficiently low temperatures: ${ }^{3-5}$ a reactive state (with high oxygen and low CO coverage) coexists with inactive state (with high CO and low oxygen coverage) for a range of CO-partial pressures (bordered by saddle-node bifurcations). For this system, there also exist waves of transition between the reactive and inactive states, where the more stable state displaces the less stable one. ${ }^{5}$ Other behavior observed in surface reactions includes reactive pulses in excitable systems, and waves of transition into unstable states. ${ }^{2}$

Even in the earliest studies, ${ }^{3}$ it was recognized that strong interactions between reacting adspecies produce ordering or islanding, limiting the validity of the simple MF description of kinetics. Interactions can significantly modify reactivity, particularly if they induce islanding with reaction localized at island peripheries; they also produce a nontrivial coverage dependence to desorption rates. Limited mobility of some adspecies also inhibits randomization (if interactions are weak) or equilibration (more generally), thus also affecting reactivity. Given this understanding of the influence of 
interactions on kinetics, it is common to account for these effects at least approximately in phenomenological rate equations. ${ }^{1}$

In contrast, relatively little attention has been paid to the description of surface diffusion in the RDEs beyond the default Laplacian formulation, where the diffusion of each adspecies is treated independently with constant (possibly anisotropic) diffusion coefficients. ${ }^{2,6}$ However, for "dense" adlayers involving even just a single adspecies, it is well known that interactions produce a complex coveragedependence to chemical diffusion. ${ }^{7}$ The same is true for mixed adlayers of relevance for reactions, but here there are significant additional complications due to the interference on diffusion of an adspecies by the presence of coadsorbed species. This results in a coupling between concentration (i.e., coverage) gradients and diffusive fluxes for distinct species, and thus produces a tensorial form of the diffusion coefficients. $^{8-11}$

Clearly, the presence of interactions by modifying diffusivity will influence the structure of chemical waves or reaction fronts. ${ }^{12,13}$ The specific feature that attractive interactions and associated phase separation can produce "sharp" interfaces in reaction fronts ${ }^{13}$ (somewhat akin to phase waves described by the Cahn-Hilliard or Allen-Cahn equations) has prompted recent application of mesoscale modeling to pattern formation in surface reactions. Here, one starts from master equations for various configurations probabilities, and then reduces these to stochastic Langevin-type rate equations subject to the key assumption of complete mixing on short length scales. ${ }^{14}$ This approach necessarily incorporates the effect of interactions into the kinetics and diffusion, albeit in a MF fashion, and thus provides the possibility to explore a variety of nanoscale pattern formations. ${ }^{14}$ However, it does not provide an exact treatment of the effects of local ordering due to strong short-ranged interactions.

Atomistic lattice-gas (LG) models do provide a formalism with which to describe precisely ordering or islanding induced by such interactions, and to treat the nontrivial nature of chemical diffusion (at least for chemisorption systems where adsorption sites are localized). Historically, LG modeling has been successfully applied to provide detailed insight into ordering and phase transitions in equilibrated adlayers. ${ }^{15}$ LG models have also been applied to nonequilibrium surface reaction type models, in many cases inspired by the Ziff-Gulari-Barshad (ZGB) model. ${ }^{16}$ In equilibrium, the actual values of rates for atomistic processes are not important, only the detailed-balance constraint relating rates for forward and reverse processes. For surface reaction models, one must of course incorporate the appropriate adsorption, desorption, and reaction steps [where reaction occurs between adsorbed species in the Langmuir-Hinshelwood (LH) type mechanisms of interest here]. However, to correctly describe the kinetics and fluctuations in nonequilibrium phenomena, LG models must also be implemented with the appropriate values of rates for all relevant atomistic processes (including diffusion) which change the occupancy of sites on the lattice. Clearly, rapid diffusion causes local equilibration, which impacts reaction kinetics. At least as significant is the more subtle feature that rapid surface diffusion is responsible for the strong hysteresis observed in experiments where the LH mechanism produces bistability. ${ }^{17,18}$ Most LG models have been implemented without surface diffusion, or with diffusion rates on the order of other rates, and thus do not address the relevant hydrodynamic regime. One exception is where behavior with increasing hop rates is studied systematically and extrapolated to very large hop rates. ${ }^{9,10,18,19}$ The inadequacies of limited mobility models are also immediately evident when describing chemical wave phenomena (see the following).

However, LG modeling is the natural approach to precisely describe surface reactions provided it includes the following ingredients: the appropriate LH mechanism; incorporation of realistic adspecies interactions in specifying rates for various atomistic processes; and effective treatment of the hydrodynamic regime of rapid diffusion. Inclusion of reliable adspecies interactions is becoming increasingly viable by exploiting results from electronic structure calculations (as well as from experiment). There are several examples of such modeling, ${ }^{20-22}$ but here we just note some particularly successful modeling of scanning tunneling microscopy data for CO-oxidation on $\mathrm{Pt}(111) .{ }^{22}$ Regarding efficient treatment of rapid diffusion of some adspecies, a "hybrid" strategy was introduced by Silverberg and Ben-Shaul, ${ }^{23}$ wherein the completely or relatively immobile adpsecies are described by a full LG model, but the highly mobile "equilibrated" adspecies are described by a MF-type treatment. This approach was first applied to titration reactions by them and others. ${ }^{20,23}$ Later extensive application of the hybrid approach to COoxidation reactions by our group showed that MF-type bistability is recovered in hybrid treatments, ${ }^{9,11,24,25}$ a feature of primary interest here. We also note work by Zhdanov and co-workers ${ }^{21}$ and Jansen and co-workers ${ }^{26}$ emphasizing the role of rapid diffusion, and developing procedures or algorithms for its efficient treatment. The latter group discuss coarse-graining or block renormalization type approaches, but the applicability of these is limited to rather special cases (e.g., local randomization of all adsorbates). ${ }^{26}$

In this work, we primarily explore the basic features of canonical LG models for CO-oxidation with a $\mathrm{LH}$ reaction mechanism exhibiting MF bistability. Rather than fine tuning these models to incorporate interactions appropriate to specific systems, we emphasize fundamental and generic issues. Our focus in Sec. II is on the behavior of the LG model with finite hop rate for $\mathrm{CO}$ diffusion, and in particular we discuss the variation of behavior with increasing hop rate as one approaches the hydrodynamic regime. As indicated previously, we emphasize that the primary challenge in LG modeling of surface reactions is the appropriate description of the "hydrodynamic" regime of rapid surface diffusion of some reactant adspecies. In Sec. III, we develop concepts and strategies to directly treat this regime. ${ }^{9,10}$ Our primary specific goal is a "proof of principle" that exact RDEs can be obtained for LG reaction models. We show this by comparing results of LG models extrapolated for large hop rates with those obtained from our "exact" RDEs. Thus, for our simple models, we achieve the ultimate goal of exactly describing sptaiotemporal behavior in LG models in the hydrodynamic regime without approximation. To achieve this goal, it was 
necessary to characterize percolative diffusion of $\mathrm{CO}$ (ads) through a disordered environment of coadsorbed $\mathrm{O}$ (ads), as described in Sec. IV. A discussion of the rather severe simplifications and limitations of our modeling, and challenges for more realistic treatments, is presented in Sec. V. Finally, in Sec. VI, we briefly discuss other reaction systems and the associated analysis of chemical diffusion (viewed as transport with dynamic or quenched disorder depending on the relative mobility of the coadsorbed adspecies). ${ }^{9-11}$ We also present our general conclusions.

\section{CO-POISONING TRANSITIONS, METASTABILITY, AND FLUCTUATING CHEMICAL WAVES IN LATTICE-GAS MODELS FOR CO-OXIDATION}

An example of a $\mathrm{LH}$ reaction mechanism producing bistability in the hydrodynamic regime is provided by $\mathrm{CO}$ oxidation on surfaces without substrate reconstruction or other feedback mechanisms. ${ }^{1-3}$ We now describe the simplified models considered in this work, where $\mathrm{CO}$ (ads) and $\mathrm{O}$ (ads) reside on a common square lattice of adsorption sites, and coverages in monolayers are denoted by $\theta_{\mathrm{CO}}$ or $\theta_{\mathrm{O}}$, respectively. In the following, "gas" denotes gas phase, and "ads" denotes adsorbed phase. The LH mechanism involves the following steps:

(i) adsorption of $\mathrm{CO}$ (gas) at single empty sites at rate $P_{\mathrm{CO}}$ (per site), and desorption at rate $d$;

(ii) random hopping of $\mathrm{CO}$ (ads) to nearby empty sites with some characteristic rate $h,^{27}$

(iii) dissociative adsorption of $\mathrm{O}_{2}$ (gas) with impingement rate $P_{\mathrm{O}_{2}}$ (per site) at suitable pairs of empty sites (described in more detail in the following), and possible limited mobility of $\mathrm{O}(\mathrm{ads})$;

(iv) reaction of adjacent pairs of $\mathrm{CO}(\mathrm{ads})$ and $\mathrm{O}$ (ads) at rate $k$ (per pair).

We do wish to emphasize that the adoption of a common adsorption site for $\mathrm{CO}(\mathrm{ads})$ and $\mathrm{O}$ (ads), and the neglect of $\mathrm{CO}(\mathrm{ads})-\mathrm{O}$ (ads) and $\mathrm{CO}(\mathrm{ads})-\mathrm{CO}(\mathrm{ads})$ interactions makes these models highly idealized. These features and their effect on model behavior are discussed in Sec. V.

The standard choice in these idealized models is for adsorption of $\mathrm{O}_{2}$ (gas) onto adjacent or nearest-neighbor (NN) empty sites. ${ }^{9,16-19,24,28}$ If $\mathrm{O}(\mathrm{ads})$ mobility is included, then it would involve random hopping to adjacent empty sites. One shortcoming of this model is an unphysical poisoning transition to an oxygen-covered surface, for sufficiently high $P_{\mathrm{O}_{2}} / P_{\mathrm{CO}} \cdot{ }^{16}$ Thus, we shall also consider a modified model where $\mathrm{O}_{2}$ (gas) adsorbs at diagonally adjacent empty sites, provided the six additional neighbors are also free of $\mathrm{O}$ (ads). ${ }^{21,25,29,30}$ See Fig. 1. This is termed the "eight-site rule" since an ensemble of eight sites must be free of $\mathrm{O}$ (ads) for adsorption to occur, and it reflects very strong NN repulsive $\mathrm{O}(\mathrm{ads})-\mathrm{O}$ (ads) interactions. ${ }^{31}$ Any $\mathrm{O}$ (ads) mobility must be consistent with these interactions, so $\mathrm{O}$ (ads) hops only to adjacent empty sites with no $\mathrm{O}(\mathrm{ads})$ neighbors. ${ }^{30}$ In the modified model, no $\mathrm{NN}$ pairs of $\mathrm{O}$ (ads) can be created, so $\theta_{\mathrm{O}} \leqslant 1 / 2$, which precludes oxygen poisoning.

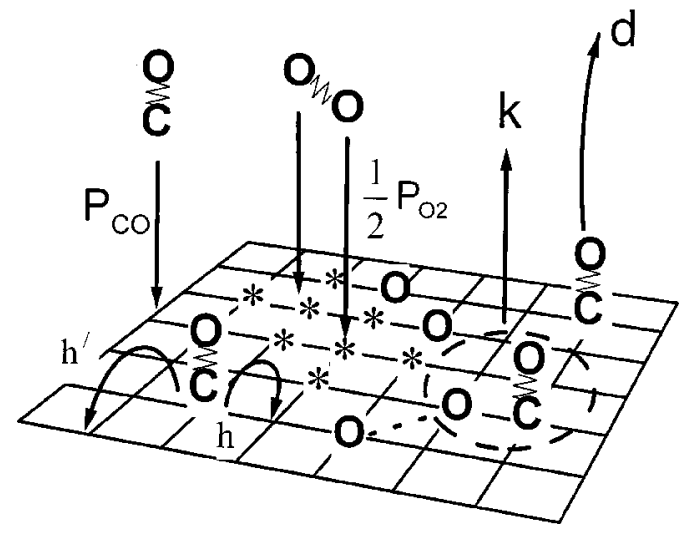

FIG. 1. Schematic of our modified lattice-gas reaction model for COoxidation (Refs. 25 and 29). Dissociative adsorption of $\mathrm{O}_{2}$ (gas) is indicated with impingement rate $\frac{1}{2} P_{\mathrm{O}_{2}}$ per diagonal pair of sites (or $P_{\mathrm{O}_{2}}$ per site, since there are two diagonal pairs per site). Sites denoted by an asterisk are required to be free of $\mathrm{O}$ (ads) for $\mathrm{O}_{2}$ (gas) adsorption according to the eight-site rule (and the central two asterisk sites must also be empty). $\mathrm{CO}$ (gas) adsorption with impingement rate $P_{\mathrm{CO}}$ per site, $\mathrm{CO}(\mathrm{ads})$ desorption at rate $d$, and reaction of adjacent $\mathrm{CO}(\mathrm{ads})$ and $\mathrm{O}(\mathrm{ads})$ at rate $k$, are also indicated. Hopping of $\mathrm{CO}$ (ads) to $\mathrm{NN}$ empty sites occurs at rate $h$, and to next NN empty sites at rate $h^{\prime}$. Typically, we expect that $h \gg h^{\prime} \gg P_{\mathrm{CO}}+P_{\mathrm{O}_{2}}($ or $k$ or $d)$.

We emphasize, however, that apart from the artificial oxygen poisoning transition in the standard model, the basic behavior for both models is similar for small $d$ as regards the transition to a (nearly) CO-poisoned state for sufficiently high $P_{\mathrm{CO}} / P_{\mathrm{O}_{2}}$, and associated chemical wave propagation discussed in the following. In particular, this is true for the somewhat artificial case $d=0$ where the completely COcovered surface is an inactive steady state. ${ }^{32}$ Thus, the following discussion applies for both models, unless otherwise indicated, and often gives specific results for $d=0$.

In our analysis of LG reaction models that follows we choose the time scale so that the impingement rates satisfy $P_{\mathrm{CO}}+P_{\mathrm{O}_{2}}=1$. We primarily consider the case $k=1$ (but see Appendix A for $k=\infty$ ), and focus on the regime of small $d$. It is also natural to define a characteristic length, ${ }^{18} L_{C}=\left(L_{R}^{2}\right.$ $\left.+L_{D}^{2}\right)^{1 / 2}$, in terms of a spatial coupling range, $L_{R}$, and a diffusion length, $L_{D}$. Here, $L_{R}$ denotes the range of the "direct spatial coupling" due to reaction or molecular adsorption on distinct (nearby) sites, and thus is of the order of the surface lattice constant, $a .^{18}$ The diffusion length satisfies ${ }^{33}$ $L_{D}=(D / K)^{1 / 2}$, where $D \sim a^{2} h$ indicates the typical value of the dominant diffusion coefficient for the mobile species in terms of its hop rate, $h$. Here, $K$ is an effective overall firstorder rate for the reaction. The relevant hydrodynamic regime corresponds to $L_{C} \approx L_{D}=(D / K)^{1 / 2} \gg a$. Also, if $L$ denotes linear system size, in the following we consider only "large" linear system sizes $L \gg L_{C}$. Appendix B briefly treats the case where $L \ll L_{C}$, which is of relevance in the study of fluctuation effects in nanoscale reaction systems. ${ }^{29}$

\section{A. Steady states in spatially uniform systems}

For small $d$, the above-mentioned LG models with finite rate, $h$, of $\mathrm{CO}$ hops to $\mathrm{NN}$ empty sites typically ${ }^{34}$ exhibit a discontinuous or first-order kinetic phase transition at some $P_{\mathrm{CO}}=P^{*}(h)$ between a "reactive" stable steady-state (for 

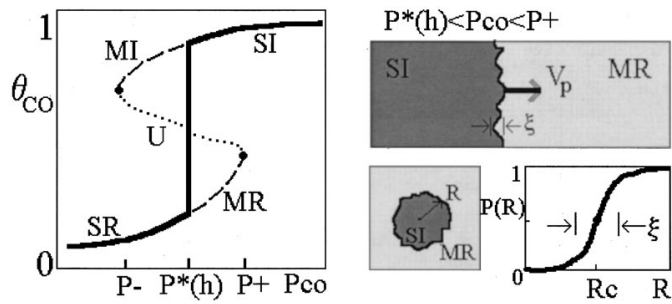

FIG. 2. Schematic of the steady-state phase diagram for our lattice-gas models of CO-oxidation for finite $h$ showing $\theta_{\mathrm{CO}}$ vs $P_{\mathrm{CO}}$. Solid curves indicating the stable reactive (SR) [stable inactive (SI)] steady states for $P_{\mathrm{CO}}<P^{*}(h)\left[\right.$ for $\left.P_{\mathrm{CO}}>P^{*}(h)\right]$ are separated by a discontinuous transition at $P_{\mathrm{CO}}=P^{*}(h)$. Metastable reactive (MR) and inactive (MI) extensions are denoted by dashed lines (the extent of which increases dramatically with increasing $h$ ), and an ill-defined unstable state $(U)$, for finite $h$, is denoted by a dotted line. Schematics on the right-hand side indicate a blob of the stable inactive state embedded in the metastable reactive state for $P_{\mathrm{CO}}>P^{*}$, and the associated growth probability $P(R)$ vs radius, $R$. Also shown schematically is planar wave of propagation of the stable inactive state into the metastable reactive state for $P_{\mathrm{CO}}>P^{*}$.

$\left.P_{\mathrm{CO}}<P^{*}\right)$ and an "inactive" stable steady state (for $P_{\mathrm{CO}}$ $\left.>P^{*}\right) .{ }^{16,18,19,28,35}$ The reactive state has high $\theta_{\mathrm{O}}$ and low $\theta_{\mathrm{CO}}$ (and high $\mathrm{CO}_{2}$ production), whereas the situation is reversed for the inactive state. The reactive state has a metastable extension for $P^{*}<P_{\mathrm{CO}}<P_{+}(h)$, and the inactive state has such an extension for $P_{-}(h)<P_{\mathrm{CO}}<P^{*}$, where $P_{+}$and $P_{-}$ correspond to an upper and lower spinodals, respectively. These $P_{ \pm}$become saddle-node bifurcations as $h \rightarrow \infty$. See Fig. 2. For small $h$, metastability or hysteresis about this transition is very weak in that the width of the metastable region $\left(P^{+}-P^{-}\right)$is negligible compared to the width of the bistable region in the corresponding MF rate equations. ${ }^{17,18,36}$ We note however that the lifetime of metastable states still diverges approaching the transition, $\Delta$ $=P_{\mathrm{CO}} P^{*}(h) \rightarrow 0$. This weak metastability is due to "large fluctuations" associated with the adsorption and reaction processes. Introducing significant diffusion (as in real systems) quenches these fluctuations, dramatically expanding the range of metastability, and ultimately producing bistability (metastable state lifetimes become infinite), as $h \rightarrow \infty .{ }^{17,18,24}$ This "hydrodynamic" regime recovers the hysteresis behavior of experimental reaction systems. The discontinuous transition for finite $h$ becomes the equistability point in the bistable system for $h \rightarrow \infty .{ }^{9,17,37}$ See Sec. II B.

One significant feature suggested by our simulation results is that

$$
\begin{aligned}
P^{*}(h) \sim & P^{*}(\infty)+A / L_{C}, \\
& \text { or that } P^{*}(h) \sim P^{*}(\infty)+B / h^{1 / 2} \text { as } h \rightarrow \infty .
\end{aligned}
$$

The origin of this behavior might reflect shifts with increasing $h$ in steady-state coverages due to "mixing" over a greater length $\sim L_{C}$, or subtle changes in percolative transport of $\mathrm{CO}$ (ads) (see Sec. II B) ${ }^{38}$ A more detailed discussion will be presented elsewhere. ${ }^{34}$

Next, we briefly discuss "nucleation theory" for these first-order transitions. ${ }^{18}$ For $P_{-}<P_{\mathrm{CO}}<P_{+}$, consider a blob of the stable phase of radius $R$ embedded in the metastable phase. The probability, $\mathrm{P}(R)$, of growth and survival of the blob (versus shrinkage and death) increases with $R$, and satisfies $\mathrm{P}(0)=0$ and $\mathrm{P}(\infty)=1$. Define a critical radius $R$ $=R_{c}$ so that $\mathrm{P}\left(R_{c}\right)=1 / 2 .{ }^{39}$ See Fig. 2. Then, we find that $R_{c} \sim L_{c} \Delta^{\sigma-1}$ diverges upon approaching transition, where $0<\sigma<1$ for small $h$, contrasting MF behavior where $\sigma$ $=0 .{ }^{18,33,35}$ Of course, the divergence of the critical size produces the divergence of the lifetime of the metastable state, mentioned previously. ${ }^{40}$ As $h \rightarrow \infty$, we find that $\sigma \rightarrow 0$ recovering MF RDE results. Concepts in Sec. II B elucidate the meaning of $\sigma$, and clarify the extent of smoothing or smearing observed in simulation data ${ }^{18}$ for $\mathrm{P}(R)$ from the deterministic step-function form $H\left(R-R_{c}\right)$.

Finally, we note that the first-order transition disappears as $d$ increases above some critical value $d=d_{c} \cdot{ }^{25,37,41-44} \mathrm{Be}-$ havior as $d \rightarrow d_{c}$ is analogous to a thermodynamic critical point with an increase in the amplitude of fluctuations. ${ }^{29,42-44}$ This critical point corresponds to a cusp bifurcation when $h \rightarrow \infty$.

\section{B. Fluctuating chemical waves in spatially nonuniform systems}

For small $d<d_{c}$ and $P_{\mathrm{CO}}$ inside the metastable region, one can construct a spatially nonuniform system with the inactive state on the left, and the reactive state on the right, say, separated by an initially sharp planar interface. The stable state always displaces the metastable state creating a planar trigger wave (so, e.g., the inactive state displaces the reactive state for $P_{\mathrm{CO}}>P^{*}$, as in Fig. 2), ${ }^{9,16,18,19,37}$ at least until the metastable state breaks down. ${ }^{39}$ The shape of the profile of the planar trigger wave is selected exponentially quickly in time with a width scaling like $L_{C} \cdot{ }^{45}$ The propagation velocity of the planar wave has the form ${ }^{9,18,19,37}$

$$
V_{p} \approx c K L_{C}\left[P_{\mathrm{CO}}-P^{*}(h)\right]=c K L_{C} \Delta,
$$

near the transition, for some constant $c$, and where again $K$ is an effective overall first-order rate. Thus, a stable stationary interface is formed between the two equistable states at $P_{\mathrm{CO}}=P^{*}(h)$. See Fig. 3 .

Combining expression (1) in Sec. II A for $P^{*}(h)$ with Eq. (2) for $V_{p}$ above yields the interesting result that

$$
\begin{aligned}
V_{p} \approx & V_{p}^{*}+c K L_{C}\left[P_{\mathrm{CO}}-P^{*}(\infty)\right], \\
& \text { where } V_{p}^{*} \approx-c K A \text { is independent of } h .
\end{aligned}
$$

The implied "near-crossing" of the family of curves for $V_{p}$ vs $P_{\mathrm{CO}}$ (for various $h$ ) at the point $\left(V_{p}, P_{\mathrm{CO}}\right)$ $=\left(V_{p}^{*}, P^{*}(\infty)\right)$ has in fact been observed previously in simulation studies (see Fig. 3),,${ }^{9,197}$ but was not elucidated. This crossing often seems quite well satisfied, even for small $h$, providing a convenient way to estimate $P^{*}(\infty)$ from such data. ${ }^{9,37}$ Given the practical relevance of the hydrodynamic limit $h \rightarrow \infty$, this is a particularly useful feature.

Significant fluctuations occur at the trigger wave front for finite $h$ (Fig. 3), and these produce the smearing in $\mathrm{P}(R)$ mentioned in Sec. II A. ${ }^{46}$ Analogous to interfaces in fluids, the rms amplitude of these fluctuations, $\xi$, is decomposed as $\xi^{2}=\xi_{i}^{2}+\xi_{0}^{2} \cdot{ }^{9,18}$ Here, $\xi_{i}$ denotes the amplitude of "intrinsic fluctuations" which are large for small $h$. For long times, $\xi$ is 


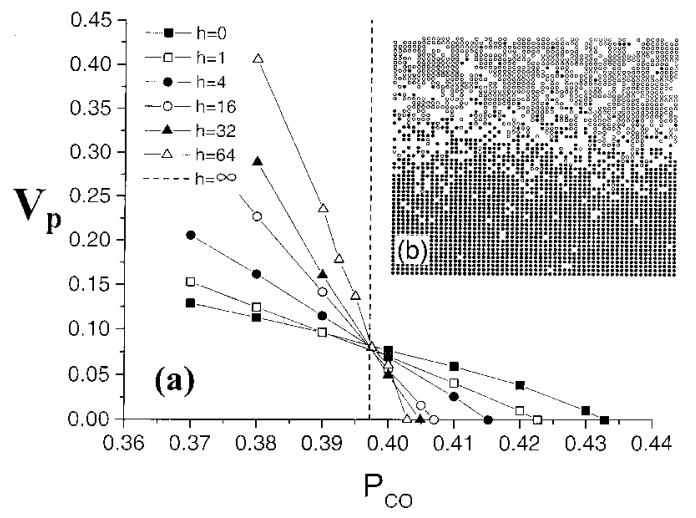

FIG. 3. (a) Propagation velocity, $V_{p}$, of a planar chemical wave versus $P_{\mathrm{CO}}$ in the standard model for CO-oxidation with $k=1$ and $d=0$ (Ref. 9). Curves for $V_{p}$ vs $P_{\mathrm{CO}}$ and different $h$ values (shown) display a near-crossing phenomenon discussed in the text. (b) Simulated interface between the reactive state (top) and inactive state (bottom) of the stationary chemical wave for $d=0$ and $h=16$ at the discontinuous transition, $P_{\mathrm{CO}}=P^{*}(h=16)$ (Ref. 9). A closed circle denotes $\mathrm{CO}$ (ads) and an open circle denotes $\mathrm{O}$ (ads).

dominated by long-wavelength fluctuations, $\xi_{0} \cdot{ }^{46}$ These are described by the stochastic Kardar-Parisi-Zhang (KPZ) equation, since the mean propagation velocity is largely independent of the direction of propagation. ${ }^{18,19}$ For KPZ evolution, one finds a dependence of the interface velocity, $V$, on front curvature, $\kappa$, of the form $\delta V=V-V_{p} \approx \nu \kappa$ (where again $V_{p}$ is the velocity of the planar interface). Here, $\nu$ is a "kinetic surface tension" acting to reduce fluctuations. Thus, the velocity of a curved blob of radius $R$ satisfies $^{18}$

$V(R) \sim V_{p}-\nu / R$,

so $V\left(R_{c}\right)=0$ for the critical radius gives $R_{c}=\nu / V_{p}$.

For consistency with the result $R_{c} \sim L_{c} \Delta^{\sigma-1}$ in Sec. II A, one has $\nu \sim K L_{c}^{2} \Delta^{\sigma}$, where $0<\sigma<1$ for small $h$. Thus, for finite $h, \nu$ vanishes at $P_{\mathrm{CO}}=P^{*}$ leading to "strongly fluctuating" stationary interfaces. ${ }^{18,19}$ Since $\sigma \rightarrow 0$ for large $h$, so one obtains $\nu \sim h$ in the hydrodynamic regime, consistent with standard results for MF RDEs. ${ }^{33}$

\section{DIRECT ANALYSIS OF THE HYDRODYNAMIC REGIME FOR CO-OXIDATION: HYBRID MODELS, PARALLEL ALGORITHMS FOR NONUNIFORM SYSTEMS}

Our central goal is to develop procedures for direct and precise analysis of the hydrodynamic regime, where our COoxidation models are described by exact RDEs of the form $^{9,37}$

$$
\begin{aligned}
& \partial / \partial t \theta_{\mathrm{CO}}=P_{\mathrm{CO}} \theta_{E}-R_{\mathrm{CO}_{2}}-d \theta_{\mathrm{CO}}-\nabla \cdot \mathbf{J}_{\mathrm{CO}}, \\
& \partial / \partial t \theta_{\mathrm{O}}=2 P_{\mathrm{O}_{2}} S_{\mathrm{O}_{2}}-R_{\mathrm{CO}_{2}} .
\end{aligned}
$$

The meaning of the different terms describing changes due to adsorption, reaction, desorption, or diffusion will be clear from the following definitions: $\theta_{E}=1-\theta_{\mathrm{CO}}-\theta_{\mathrm{O}}$ denotes the coverage of empty sites; $S_{\mathrm{O}_{2}}$ is the normalized sticking probability for $\mathrm{O}_{2}$ (gas); $R_{\mathrm{CO}_{2}}$ the reaction rate; and $\mathbf{J}_{\mathrm{CO}}$ is the diffusive flux for $\mathrm{CO}$ (ads). There is no diffusive flux term for $\mathrm{O}$ (ads) as its mobility is negligible relative to that of
$\mathrm{CO}$ (ads). For our models with no interactions between $\mathrm{CO}$ (ads) and other $\mathrm{CO}$ (ads) or $\mathrm{O}$ (ads) (other than site blocking due to the feature that in our models, these adspecies share a common adsorption site), one has that ${ }^{9,11}$

$$
\begin{aligned}
\mathbf{J}_{\mathrm{CO}}= & -D_{\mathrm{CO}, \mathrm{CO}} \nabla \theta_{\mathrm{CO}}-D_{\mathrm{CO}, \mathrm{O}} \nabla \theta_{\mathrm{O}}, \\
& \text { where } D_{\mathrm{CO}, \mathrm{O}}=D_{\mathrm{CO}, \mathrm{CO}} \theta_{\mathrm{CO}} /\left(1-\theta_{\mathrm{O}}\right) .
\end{aligned}
$$

The latter important relationship between $D_{\mathrm{CO}, \mathrm{O}}$ and $D_{\mathrm{CO}, \mathrm{CO}}$ actually appeared earlier in thermodynamic ${ }^{47}$ and kinetic ${ }^{17,24}$ treatments of diffusion in noninteracting mixed overlayers, however these treatments produced incorrect values for the individual coefficients (see Sec. IV and Appendix C for a correct analysis). This result explicitly reveals coupling of the diffusive flux for $\mathrm{CO}$ (ads) to gradients in the concentration of $\mathrm{O}$ (ads), and ensures that $\mathbf{J}_{\mathrm{CO}} \rightarrow \mathbf{0}$, for a "jammed" surface, where $\theta_{\mathrm{CO}}+\theta_{\mathrm{O}} \rightarrow 1$.

For the standard model, one has $R_{\mathrm{CO}_{2}}=4 k \theta_{\mathrm{O} \cdot \mathrm{CO}}$, where $\theta_{\mathrm{O} . \mathrm{CO}}$ is the concentration of adjacent pairs of $\mathrm{CO}(\mathrm{ads})$ and $\mathrm{O}$ (ads), and $S_{\mathrm{O}_{2}}=\theta_{E \cdot E}$ is the concentration of adjacent pairs of empty sites. For the modified model, one has the exact relation

$$
R_{\mathrm{CO}_{2}}=4 k \theta_{\mathrm{O}} \theta_{\mathrm{CO}}^{\mathrm{loc}},
$$

where $\theta_{\mathrm{CO}}^{\text {loc }}=\theta_{\mathrm{CO}} /\left(1-\theta_{\mathrm{O}}\right)$ is the local coverage of $\mathrm{CO}$ (ads), ${ }^{25}$ and $S_{\mathrm{O}_{2}}$ now denotes the probability of finding an "adsorption ensemble" of two diagonally adjacent empty sites surrounded by six sites not occupied by $\mathrm{O}(\mathrm{ads}){ }^{25}$ The exact result for $R_{\mathrm{CO}_{2}}$ in the modified model follows since each $\mathrm{O}$ (ads) has four neighboring sites which are equally likely to be populated by the "randomly" distributed $\mathrm{CO}$ (ads). However, the other quantities cannot be simply expressed in terms of adspecies coverages due to nontrivial spatial correlations, so Eq. (5) is not closed but can be thought of as the lowest order equations in an infinite hierarchy.

We shall consider solutions to Eq. (5) (and this hierarchy) which correspond to planar wave fronts propagating in the $x$ direction (so $\nabla=\partial / \partial x$ ), and which thus have the form

$$
\theta_{\mathrm{CO}}=\theta_{\mathrm{CO}}\left(x-V_{p} t\right), \quad \theta_{\mathrm{O}}=\theta_{\mathrm{O}}\left(x-V_{p} t\right),
$$

and $\theta_{\mathrm{O} \cdot \mathrm{CO}}=\theta_{\mathrm{O} \cdot \mathrm{CO}}\left(x-V_{p} t\right)$ for the standard model, etc. This requires both a correct treatment of the non-MF kinetics, and a precise analysis of the problem of percolative diffusion of $\mathrm{CO}$ (ads) through a "quenched" disordered environment of coadsorbed $\mathrm{O}$ (ads). The latter results in a decrease to zero of $D_{\mathrm{CO}, \mathrm{CO}}=D_{\mathrm{CO} \cdot \mathrm{CO}}\left(\left\{\theta_{\mathrm{O}}\right\}\right)$ as $\theta_{\mathrm{O}}$ increases to some percolation threshold. Here, $\left\{\theta_{\mathrm{O}}\right\}$ indicates a dependence on the actual distribution of $\mathrm{O}(\mathrm{ads})$, rather than just on $\theta_{\mathrm{O}} \cdot{ }^{9}$ See Sec. IV.

\section{A. Analysis of non-MF kinetics and steady states}

To analyze directly the non-MF kinetics and steady states for $h \rightarrow \infty$, we implement a "hybrid" simulation model..$^{9,24,25,29,30}$ The $\mathrm{O}$ (ads) distribution is described by appropriate LG model simulations. However, if $\mathrm{CO}(\mathrm{ads})$ is randomly distributed on non-O(ads) sites due to rapid mobility (and no interactions), it suffices to track only the number of $\mathrm{CO}$ (ads), e.g., using a MF parameter, $\theta_{\mathrm{CO}}$. Actually, there is a slight disconnect between this hybrid model and our LG 

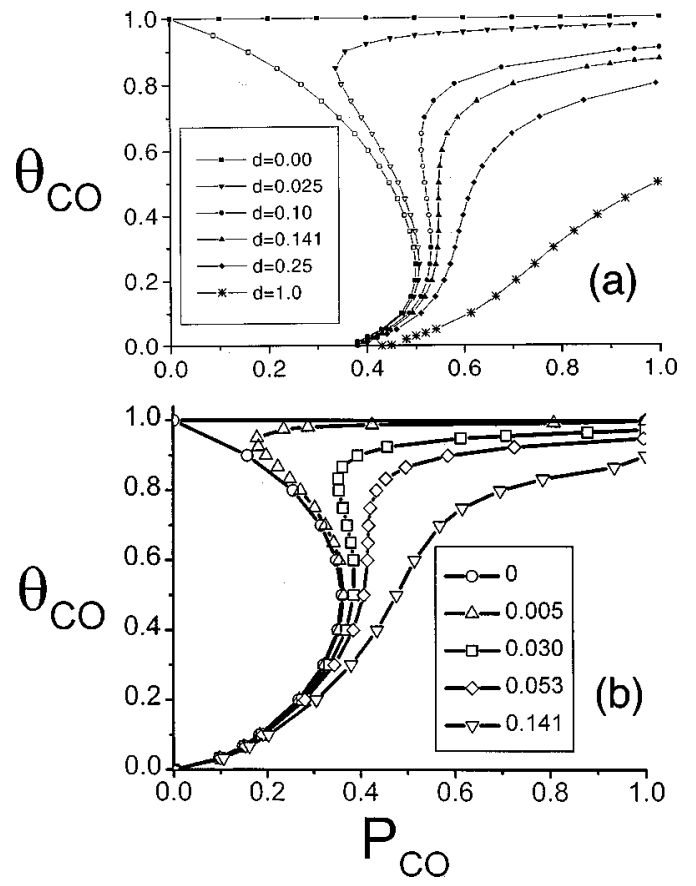

FIG. 4. Steady-state phase diagrams for $\theta_{\mathrm{CO}}$ vs $P_{\mathrm{CO}}$ from hybrid model simulations (where $h \rightarrow \infty$ ) with $k=1$ for: (a) the standard model (Ref. 24); (b) the modified model (Ref. 25). Behavior for various $d$ (shown) reveals the disappearance of bistability above a critical $d=d_{c}$.

models with $\mathrm{CO}$ hopping to $\mathrm{NN}$ sites at rate $h$. In the $\mathrm{LG}$ model, a $\mathrm{CO}$ (ads) cannot necessarily reach all non-O(ads) sites by $\mathrm{NN}$ hopping, so different local $\mathrm{CO}$ coverages could exist in disconnected non-O(ads) regions (at least for higher $\left.\theta_{\mathrm{O}}\right)$. But in practice this effect is negligible.

As noted in Sec. I, this hybrid approach was first introduced to treat the titration of preadsorbed $\mathrm{O}(\mathrm{ads})$ by exposure to $\mathrm{CO}$ (gas) to form $\mathrm{CO}_{2}$ (gas). ${ }^{23}$ However, the benefits of this approach are even more dramatic in our analysis of CO-oxidation models! Specifically, hybrid simulations immediately reveal true bistability of the CO-oxidation model in the hydrodynamic regime, ${ }^{24,25}$ entirely analogous to behavior in standard MF treatments.

Conventional simulations of the hybrid model allow complete characterization of the stable steady states, but not the unstable steady states. ${ }^{24,25}$ In contrast, both stable and unstable steady states can be immediately determined from analysis of rate equations in MF treatments. Fortunately, we can utilize a novel constant-coverage simulation procedure ${ }^{48}$ to characterize unstable (as well as stable) steady states. ${ }^{24,25}$ In this approach, a target coverage, $\theta_{\mathrm{CO}}^{\mathrm{cc}}$ of $\mathrm{CO}(\mathrm{ads})$ is specified, and the adsorbing species is selected at each step to maintain $\theta_{\mathrm{CO}}^{\mathrm{cc}}$. Then, the $\mathrm{P}_{\mathrm{CO}}$ corresponding to $\theta_{\mathrm{CO}}=\theta_{\mathrm{CO}}^{\mathrm{cc}}$ is given by the resulting fraction adsorption attempts of $\mathrm{CO}$ vs $\mathrm{O}_{2}$. Thus, we can obtain complete information on steady states (as in the MF).

In Fig. 4, we compare simulation results for the steady state $\theta_{\mathrm{CO}}$ vs $P_{\mathrm{CO}}$ for both the standard and modified models with $k=1$ and immobile $\mathrm{O}$ (ads). Apart from the unphysical transition to an oxygen poisoning state for $P_{\mathrm{CO}}<0.378^{24}$ in the former, behavior is qualitatively similar. One finds that $d_{c}=0.142(0.052)$ in the standard ${ }^{24}\left(\right.$ modified $\left.^{25}\right)$ model.

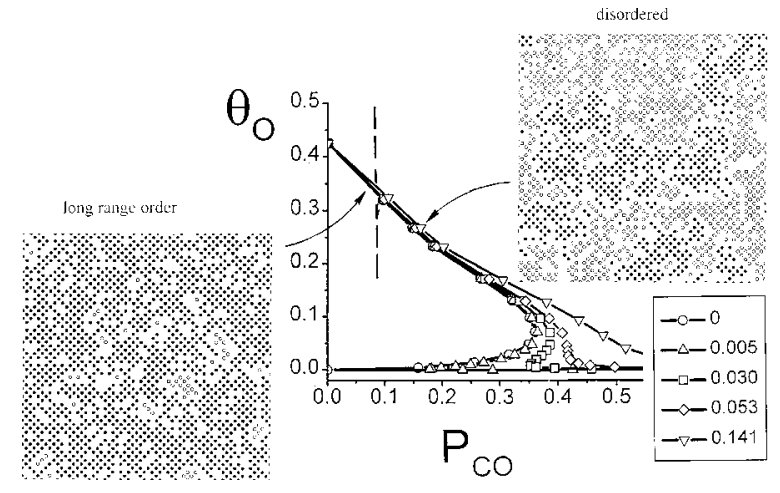

FIG. 5. Steady-state phase diagram for $\theta_{\mathrm{O}}$ vs $P_{\mathrm{CO}}$ from hybrid model simulations (where $h \rightarrow \infty$ ) for the modified model with $k=1$ and various $d$ (shown)-Ref. 25. Also indicated schematically is the symmetry-breaking transition between short-range and long-range $c(2 \times 2)$ order of $\mathrm{O}(\mathrm{ads})$ (vertical dashed line). For lower $P_{\mathrm{CO}}$ or higher $\theta_{\mathrm{O}}$, populations of the two $c(2 \times 2)$ sublattices become unequal. Here, closed and open circles both represent $\mathrm{O}$ (ads), but on the different sublattices.

Here, we just elaborate on the nature of the reactive state in the modified model for immobile $\mathrm{O}(\mathrm{ads})$ where infinite $\mathrm{NN} \mathrm{O}$ (ads) $-\mathrm{O}$ (ads) repulsions ensure $\theta_{\mathrm{O}}<1 / 2$ (so no oxygen poisoning), and result in checkerboard or $c(2 \times 2)$ ordering of $\mathrm{O}(\mathrm{ads})$. See Fig. 5. Here, one might expect behavior analogous to equilibrium systems with infinite NN repulsions, i.e., the hard-square (HS) model. ${ }^{49}$ Indeed, just as in the HS model, there is a symmetry-breaking transition from short-range to long-range $c(2 \times 2)$ order, where populations of the two degenerate $c(2 \times 2)$ sublattices become unequal. ${ }^{25,30,50}$ This occurs when $\theta_{\mathrm{O}}$ exceeds a critical value of $\theta_{\text {crit }}=0.300$ (corresponding to $P_{\mathrm{CO}}$ decreasing below 0.135 ) in the reaction model with $k=1$ and $d=0^{51}$ (versus $\theta_{\text {crit }}=0.369$ for the HS model $\left.{ }^{49}\right)$. Note that as $P_{\mathrm{CO}} \rightarrow 0$, very slow reactive removal of single $\mathrm{O}(\mathrm{ads})$ 's produces some "isolated defects" inside $c(2 \times 2)$ domains that cannot be refilled by adsorption of $\mathrm{O}_{2}$ (gas), so that $\theta_{\mathrm{O}}$ does not approach $\frac{1}{2} .{ }^{25}$ See Fig. 5. However, apart from some subtle but important technical differences (discussed in Sec. IV), the $c(2 \times 2)$ order-disorder transition is analogous to that in the HS model, and specifically in the same Ising universality class. ${ }^{30,50}$ Precise analysis of these issues exploits finite-size scaling techniques. ${ }^{30,50}$ The nonequilibrium nature of this reactive steady state derives from the feature that oxygen adsorbs as dimers but is removed as monomers (in contrast to the HS model which can be recast as a monomer adsorptiondesorption model with infinite NN repulsions).

Finally, it is instructive to note that instead of exact simulation analysis, one can also perform an approximate analysis of the exact master equations for these models. ${ }^{24,25}$ Usually, this involves some approximate treatment of shortrange spatial correlations. For the standard model, use of a pair approximation yields an additional equation for, say, the probability of $\mathrm{O}(\mathrm{ads})$ pairs, $\theta_{\mathrm{O} \cdot \mathrm{O}}$, from which other quantities in Eq. (5) can be determined [exploiting the random distribution of $\mathrm{CO}(\mathrm{ads})$ on non-O(ads) sites]. ${ }^{9,24}$ For the modified model, an analogous treatment provides an expression for $S_{\mathrm{O}_{2}}$ in Eq. (5) in terms of the adspecies coverages, ${ }^{52}$ 


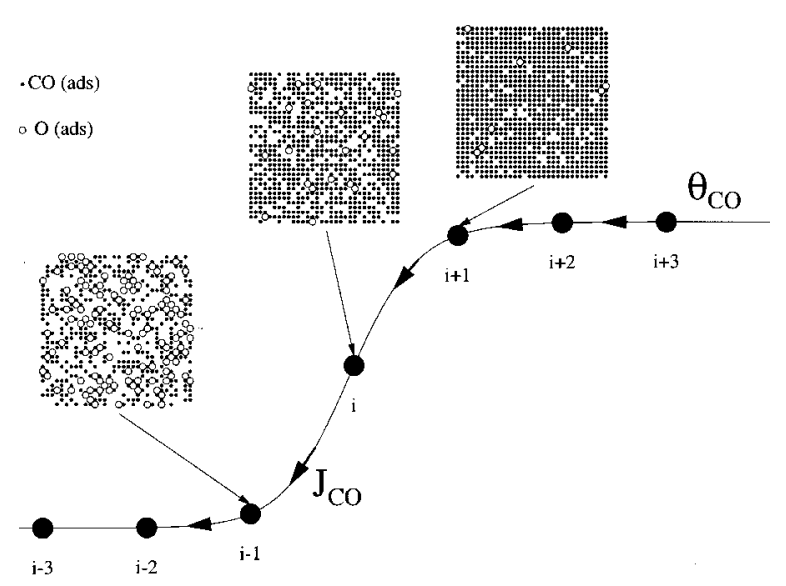

FIG. 6. Schematic indicating our parallel simulation procedure for direct analysis of chemical wave propagation in the hydrodynamic regime (Refs. 9 and 24). A discrete grid of macroscopic spatial points (labeled by ... $i-1, i$, $i+1, \ldots)$ is introduced spanning the chemical wave front. Hybrid simulations for the local nonequilibrium state of the reacting systems are performed in parallel at each of these points. These simulations are suitably coupled to reflect mass transport of $\mathrm{CO}(\mathrm{ads})$ across the surface (between these points).

so then all terms in Eq. (5) can be written in terms of $\theta_{\mathrm{CO}}$ and $\theta_{\mathrm{O}}$ closing the equations. ${ }^{25}$

\section{B. Analysis of chemical wave propagation}

To incorporate the above-mentioned precise reaction kinetics from simulation into an analysis of chemical wave propagation, we divide space into a discrete set of macroscopic points spanning the reaction front. We then perform in parallel (i.e., simultaneously) hybrid simulations at these points. The individual simulations must be suitably coupled to reflect the diffusive transport of $\mathrm{CO}$ (ads) across the surface between these points. See Refs. 9, 24, and Fig. 6. The latter coupling is nontrivial because of the complex nature of chemical diffusion. In principle, one should extract a value of $D_{\text {CO. CO }}$ (as well as the kinetics) for each macroscopic spatial point from the parallel simulations, as its value depends on the actual distribution of $\mathrm{O}(\mathrm{ads})$ in these nonequilibrium states. However, in practice, $D_{\text {CO. CO }}$ is primarily controlled by $\theta_{\mathrm{O}}$, and one can exploit results for its form presented in Sec. IV. The above-mentioned approach is analogous to the "method of lines" 53 commonly used for MF RDEs, except that here we just replace the MF rate equations integrated at each of a discrete set of spatial points with parallel hybrid simulations.

From analysis of these PDEs we can obtain the chemical wave propagation velocity, $V_{p}(h \rightarrow \infty)$ vs $P_{\mathrm{CO}}$, and thus determine $P^{*}(\infty)$ from the $P_{\mathrm{CO}}$ value where $V_{p}$ vanishes. We present results for $P^{*}(\infty)$ (Table I) and reaction front profiles at $P_{\mathrm{CO}}=P^{*}(\infty)$ (Fig. 7) for both models with $k=1, d=0$,
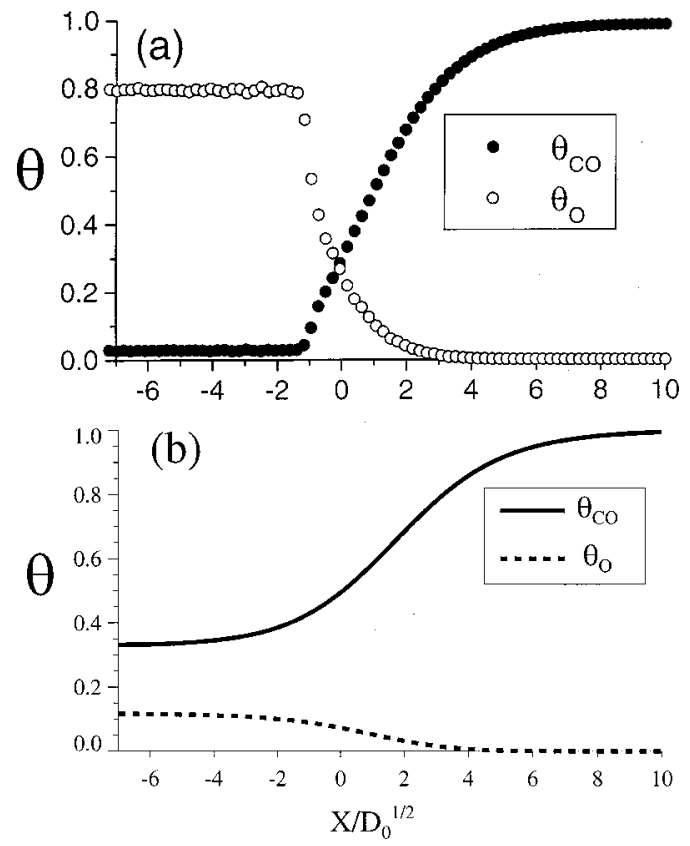

FIG. 7. Predictions for coverage profiles across a chemical wave from refined RDEs using the pair approximation to describe the reaction kinetics, and a precise description of the chemical diffusion of $\mathrm{CO}$ (ads). Results are shown at equistability for the standard (a) and modified (b) models, with $k=1, d=0$, and immobile $\mathrm{O}$ (ads).

and immobile $\mathrm{O}$ (ads). Here, we have actually used pair approximations to the reaction kinetics, rather than simulations, so that a method-of-lines treatment of the associated RDEs replaces the parallel simulations. The influence on the results of various prescriptions of diffusion was also considered in Table I. The correct treatment of diffusion for standard reaction model the (right-hand column) recovers exact behavior, $P^{*}(\infty)=0.397$ extrapolated from simulations as $h \rightarrow \infty .{ }^{54}$ This should be expected since the pair approximation adequately describes the kinetics in this case. ${ }^{9,24}$ It also recovers the unusual shape of the stationary reaction front at $P_{\mathrm{CO}}=P^{*}(\infty)$ [see Fig. $7(\mathrm{a})$ ], in contrast to the usual MF treatment. ${ }^{9}$ For the modified reaction model, we do not recover the exact result for $P^{*}(\infty)=0.325$ extrapolated from simulations as $h \rightarrow \infty .{ }^{34,55}$ However, this reflects limitations of the pair-approximation treatment of the kinetics in this case, ${ }^{25}$ rather than inadequacies in our treatment of diffusion. We shall show in a separate paper ${ }^{34}$ that an improved treatment of kinetics with our correct treatment of diffusion does recover exact behavior.

\section{CHEMICAL DIFFUSION: PERCOLATIVE TRANSPORT OF CO(ads)}

In our CO-oxidation models, there are no interactions between $\mathrm{CO}$ (ads) and other $\mathrm{CO}$ (ads) or $\mathrm{O}$ (ads). This implies

TABLE I. $P^{*}(\infty)$ for pair-approximation kinetics and various specifications of diffusivity. $D_{0}$ is a constant, $D_{\text {Co. CO }}=D\left(\theta_{0}\right)$ represents "exact" behavior (see Sec. V), and $D_{\text {Co. }} \neq 0$ comes from Eq. (2).

\begin{tabular}{lccc}
\hline \hline & $D_{\mathrm{CO} \cdot \mathrm{CO}}=D_{0}, D_{\mathrm{CO} \cdot \mathrm{O}}=0$ & $D_{\mathrm{CO} \cdot \mathrm{CO}}=D\left(\theta_{0}\right), D_{\mathrm{CO} \cdot \mathrm{O}}=0$ & $D_{\mathrm{CO} \cdot \mathrm{CO}}=D\left(\theta_{0}\right), D_{\mathrm{CO} \cdot \mathrm{O}} \neq 0$ \\
\hline Standard model & 0.4401 & 0.4084 & 0.3970 \\
Modified model & 0.3175 & 0.3146 & 0.3136 \\
\hline \hline
\end{tabular}


(a)
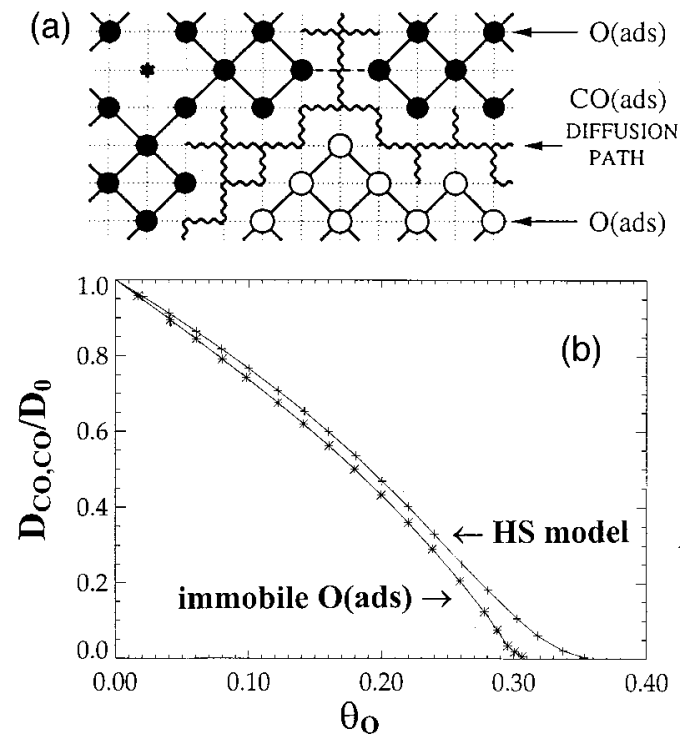

FIG. 8. (a) Schematic of diffusion paths for $\mathrm{CO}$ (ads) through coadsorbed $c(2 \times 2) \mathrm{O}(\mathrm{ads})$ in our modified model for $\mathrm{CO}$-oxidation. Closed and open circles represent $\mathrm{O}(\mathrm{ads})$, but on different $c(2 \times 2)$ sublattices. Diagonal bonds indicate $c(2 \times 2)$ clusters with next NN connectivity, which are dual to the diffusion paths for $\mathrm{CO}$ (ads). $\mathrm{CO}$ (ads) tends to percolate along antiphase boundaries for higher $\theta_{\mathrm{O}}$. (b) Simulation results for $D_{\mathrm{CO}, \mathrm{CO}}$ vs $\theta_{\mathrm{O}}$ for the modified model with immobile $\mathrm{O}(\mathrm{ads})$ and $k \gg 1$, where percolation occurs at $\theta_{\mathrm{O}}=0.305$. For contrast, we show behavior in the HS model [corresponding to a reaction model with highly mobile $\mathrm{O}$ (ads) subject to infinite $\mathrm{NN} \mathrm{O}$ (ads) $-\mathrm{O}$ (ads) repulsions], where percolation occurs at $\theta_{\mathrm{O}}=0.368$.

that the chemical diffusion coefficient, $D_{\mathrm{CO}, \mathrm{CO}}$, corresponds to the single particle diffusion coefficient (in the disordered system), ${ }^{9,11,56}$ so $D_{\mathrm{CO}, \mathrm{CO}}$ is independent of $\theta_{\mathrm{CO}}$. Thus, rather than couch the problem in terms of generic transport theory, it is more natural to exploit the language and concepts from the theory of (single particle) percolative transport in disordered systems. ${ }^{57}$ The most famous problem in the class is de Gennes' "ant-in-the-labyrinth" problem ${ }^{58}$ corresponding to the artificial case of randomly distributed $\mathrm{O}$ (ads).

If diffusion of $\mathrm{CO}(\mathrm{ads})$ is dominated by random hopping to nearest-neighbor (NN) empty sites, it is clear that the "diffusion paths" correspond to clusters of non-O(ads) sites with NN connectivity. Long-range diffusion, corresponding to $D_{\mathrm{CO}, \mathrm{CO}}>0$, requires percolation of these diffusion paths. There is a "duality" between percolation of these [NN non$\mathrm{O}(\mathrm{ads})$ cluster] diffusion paths, and percolation of clusters of $\mathrm{O}$ (ads) sites with $\mathrm{NN}$ or next NN connectivity. For the latter, $\mathrm{O}$ (ads) in the same cluster can be connected by NN or diagonal NN sites [so in the modified model, this just reduces to next $\mathrm{NN}$ connectivity for $c(2 \times 2) \mathrm{O}$ (ads) clusters]. In other words, the onset of percolation of the latter $\mathrm{NN}+$ diagonal NN O(ads) clusters coincides with the cessation of percolation of the [NN non-O(ads) cluster] diffusion paths, and thus with the vanishing of $D_{\mathrm{CO}, \mathrm{CO}} \cdot{ }^{9,19}$ See Fig. 8(a). In general, $D_{\mathrm{CO}, \mathrm{CO}}$ should decrease monotonically with increasing $\theta_{\mathrm{O}}$, and vanish at the percolation threshold, $\theta_{\mathrm{O}}=\theta_{\text {perc }}$, say. We divide the following discussion into two coverage regimes: (i) behavior for low $\theta_{\mathrm{O}}$, corresponding to diffusion of $\mathrm{CO}$ (ads) around "isolated obstacles;" (ii) critical behavior for $\theta_{\mathrm{O}} \approx \theta_{\text {perc }}$.
For low coverages, $\theta_{\mathrm{O}}$, of blocking $\mathrm{O}$ (ads) sites, it is possible to utilize Lifshitz-Stepanova-type density expansions to determine exactly the coefficients, $a_{i}$, in formal density expansions of the form ${ }^{11}$

$$
D_{\mathrm{CO}, \mathrm{CO}}=D_{0}\left(1-a_{1} \theta_{\mathrm{O}}-a_{2} \theta_{\mathrm{O}}^{2}-\cdots\right)
$$

where $D_{0}=a^{2} h$, and $a$ is the surface lattice constant. Previously, this approach was implemented by Ernst et al. ${ }^{59}$ but only for a random distribution of $\mathrm{O}(\mathrm{ads})$ sites, to obtain $a_{1}$ $=\pi-1=2.14159, a_{2} \approx 0.85571, \ldots$. In fact, these results do not apply to our CO-oxidation models, where even for very low $\mathrm{O}(\mathrm{ads})$ coverages, the O's are not randomly distributed due to the dimer nature of adsorption. For arbitrarily low $\theta_{\mathrm{O}}$, there is a mixture of dimers (created directly from adsorption) and monomers [created from the dimers by removal through reaction with a $\mathrm{CO}$ (ads) of one of the constituent $\mathrm{O}$ (ads)'s]. Thus, it is necessary to determine the influence on transport of isolated "dimer defects," as well as of isolated "monomer defects," and then to take a suitable weighted average of these effects. ${ }^{11,60}$ For the standard model, there are 1.5 times the number of monomers as dimers, and one obtains $a_{1}=2.09481 .^{11,60}$ For the modified model, there are twice as many monomers as there are (diagonal) dimers, and one obtains $a_{1}=2.55204 .^{11}$

Near percolation, and specifically for $\theta_{\mathrm{O}}$ just below $\theta_{\text {perc }}$, one expects the scaling form

$$
D_{\mathrm{CO}, \mathrm{CO}} \sim\left(\theta_{\text {perc }}-\theta_{\mathrm{O}}\right)^{\mu},
$$

where $\mu>0$ is a dynamic scaling exponent, which is somewhat dependent on the fractal dimension, $d_{f}<2$, of the diffusion paths at the percolation threshold. ${ }^{51}$ For the DeGennes problem, ${ }^{58}$ or any transport problem in the random percolation universality class where $d_{f}=91 / 49=1.8958$, the wellknown Alexander-Orbach conjecture states that $\mu=2 d_{f} / 3$ $=91 / 72=1.2639 .{ }^{57}$ This does not exactly recover the most precise numerical estimate available of $\mu=1.3101$. In the following separately discuss behavior in the standard and modified models.

\section{A. Standard model: Near-randomly distributed O(ads)}

For the standard model, although the $\mathrm{O}$ (ads) are not randomly distributed, there are only short-ranged spatial correlations. These will not affect the critical behavior near percolation, so $d_{f}$ and $\mu$ will retain the above-mentioned random percolation values. The main change is a shift (increase) in the percolation threshold to $\theta_{\text {perc }}=0.435$ (Ref. 9) from the classic random percolation value of $\theta_{\text {perc }}=1$ $-0.5927=0.4073$ for $\mathrm{NN}+$ diagonal $\mathrm{NN}$ clusters. This shift can be estimated using readily implementable (but approximate) small-cell real-space renormalization group techniques. ${ }^{9}$ In the analysis of Sec. III, we simply implement a quadratic approximation for $D_{\mathrm{CO}, \mathrm{CO}} \approx D_{0}\left(1-2.095 \theta_{\mathrm{O}}\right.$ $-\alpha \theta_{\mathrm{O}}^{2}$ ), where $\alpha$ is chosen to recover the shifted $\theta_{\text {perc }} \cdot{ }^{9}$ Of course, this approximation does not incorporate the nontrivial critical behavior with $\mu \neq 1$, but (more importantly) it 
does accurately describe the $\theta_{\mathrm{O}}$ dependence of $D_{\mathrm{CO}, \mathrm{CO}}$ over a broad range of $\theta_{\mathrm{O}}$.

\section{B. Modified model: $c(2 \times 2)$ ordering of $\mathrm{O}(\mathrm{ads})$}

In the modified model where the $\mathrm{O}(\mathrm{ads})$ exhibit $c(2$ $\times 2$ ) ordering, it is clear that for higher $\theta_{\mathrm{O}}$, transport will involve percolative diffusion along the antiphase boundaries between $c(2 \times 2)$ domains. See Fig. 8. Previously, we noted that the percolation of $c(2 \times 2)$ domains with next NN connectivity blocks long-range diffusion of $\mathrm{CO}$ (ads), and thus implies that $D_{\mathrm{CO}, \mathrm{CO}}=0$. In addition, here we observe that percolation of $c(2 \times 2)$ domains requires the occurrence of symmetry breaking, i.e., the existence of long-range $c(2$ $\times 2)$ order. This follows since percolation of a $c(2 \times 2)$ domain of one phase precludes percolation of domains of the other phase, thus implying symmetry-breaking. ${ }^{11,30}$ As a consequence, there are two possibilities: (i) $c(2 \times 2)$ percolation where $D_{\mathrm{CO}, \mathrm{CO}} \rightarrow 0$ occurs after symmetry-breaking, so $\theta_{\text {perc }}$ $>\theta_{\text {crit }}$; (ii) $c(2 \times 2)$ percolation where $D_{\mathrm{CO}, \mathrm{CO}} \rightarrow 0$ occurs simultaneously with symmetry-breaking at $\theta_{\text {perc }}=\theta_{\text {crit }}$. For (i), the correlation length is finite at $\theta_{\text {perc }}$, and random percolation criticality must apply. For (ii), the divergence of spatial correlations at $\theta_{\text {perc }}=\theta_{\text {crit }}$ suggests modified critical behavior.

For the modified model with $k=1, d=0$, and immobile $\mathrm{O}(\mathrm{ads})$, we find that scenario (i) applies with $\theta_{\text {perc }}=0.305$ vs $\theta_{\text {crit }}=0.300,{ }^{51}$ and similar behavior applies for $k=\infty .{ }^{11,30} \mathrm{In}-$ deed, the diffusion paths at the percolation threshold are found to have the random percolation $d_{f}=1.89$, and correspondingly $\mu \approx 1.3$. In contrast, introducing even a small amount of mobility of $\mathrm{O}$ (ads) (which is certainly present in reality) closes the gap between symmetry-breaking and percolation producing scenario (ii). ${ }^{11,30}$ Our previous studies incorporating substantial $\mathrm{O}(\mathrm{ads})$ mobility (i.e., analyzing HS model behavior) indicate that in this case $d_{f} \approx 1.4$ has a much lower value. This reflects the feature that the diffusion paths are quasi-one-dimensional and nearly loopless, which in turn implies that $\mu \approx d_{f} \approx 1.4 .^{30}$ The same appears true in our simulations with finite mobility shown in Fig. 9. Thus, the universality class for transport in this case is distinct from random percolation (or the ant-in-the-labyrinth problem), with $d_{f}$ perhaps corresponding to the hull (perimeter) of Ising clusters at criticality. Figures 8 and 10 contrast behavior for immobile and mobile $\mathrm{O}$ (ads). Despite the above-given detailed analysis of critical transport (and the discovery of a new universality class) for the modified model, $\theta_{\mathrm{O}}$ is typically quite low across the front of the chemical wave near equistability, and it suffices to use the form $D_{\mathrm{CO}, \mathrm{CO}}$ $\approx D_{0}\left(1-2.55204 \theta_{\mathrm{O}}\right)$ in the calculations of Sec. III.

\section{KEY ISSUES FOR MORE REALISTIC MODELS OF CO-OXIDATION}

Certainly, our models for CO-oxidation are overly simplistic. Typically, $\mathrm{CO}$ (ads) and $\mathrm{O}(\mathrm{ads})$ occupy distinct adsorption sites on Pt or Pd substrates, ${ }^{61-63}$ and in fact $\mathrm{CO}$ (ads) can populate more than one type of site at appreciable coverages. ${ }^{64}$ The feature of distinct sites for $\mathrm{CO}$ (ads) and $\mathrm{O}(\mathrm{ads})$ would tend to reduce coupling between concentration

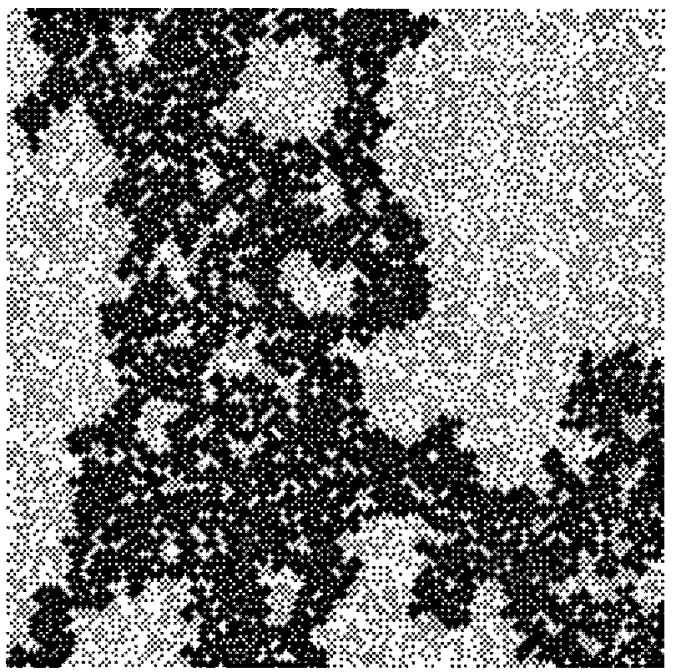

(a)

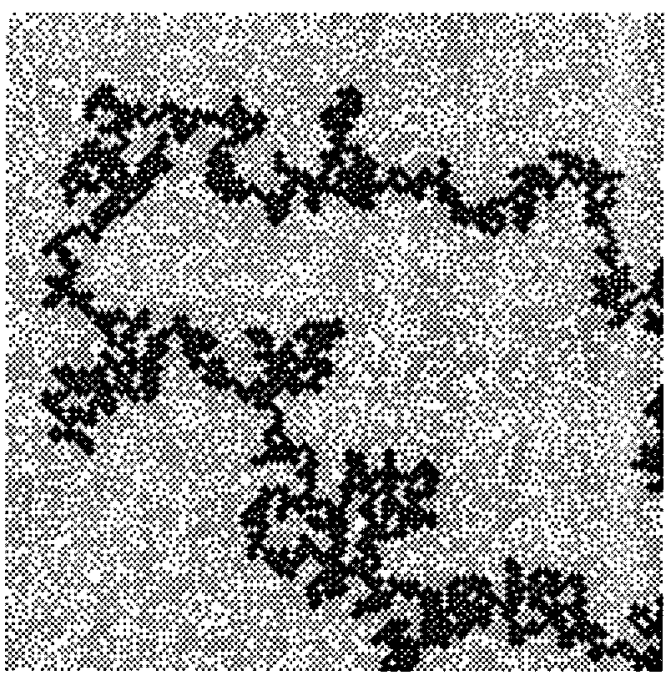

(b)

FIG. 9. Hybrid simulations of $200 \times 200$ site $\mathrm{O}$ (ads) configurations (gray) in the modified model for $\mathrm{CO}$-oxidation with $k=1$ at the percolation threshold for $c(2 \times 2)$ domains of $\mathrm{O}(\mathrm{ads})$. This threshold corresponds to the disappearance of $\mathrm{CO}$ (ads) diffusion. The diffusion path for $\mathrm{CO}$ (ads) with fractal dimension $d_{f}<2$ is shown in black. Behavior is shown for: (a) immobile $\mathrm{O}$ (ads) where percolation occurs at $\theta_{\mathrm{O}}=0.305$ (vs symmetry-breaking at $\theta_{\mathrm{O}}=0.300$ ), with $d_{f}=91 / 48=1.89$; (b) mobile $\mathrm{O}(\mathrm{ads})$ with NN hop rate of unity [hopping is subject to infinite $\mathrm{NN} \mathrm{O}(\mathrm{ads})-\mathrm{O}(\mathrm{ads})$ repulsions] where percolation and symmetry-breaking occur at $\theta_{\mathrm{O}} \approx 0.37$ (and $P_{\mathrm{CO}} \approx 0.036$ ), with $d_{f} \approx 1.4$.

gradients and diffusive fluxes for different species. Indeed, typically this coupling is neglected in MF or mesoscale modeling. ${ }^{2,14}$ However, there are also additional adspecies interactions neglected in our modeling, in particular $\mathrm{CO}$ (ads)-O(ads) repulsions, ${ }^{3,22}$ which would tend to recover this coupling. Thus, the percolative nature of CO-diffusion in our models with common adsorption sites [and no $\mathrm{CO}$ (ads)$\mathrm{O}(\mathrm{ads})$ repulsions] will likely mimic behavior in real systems with distinct adsorption sites and repulsive $\mathrm{CO}(\mathrm{ads})-\mathrm{O}$ (ads) interactions.

The presence of $\mathrm{CO}($ ads $)-\mathrm{O}$ (ads) and $\mathrm{CO}($ ads $)-\mathrm{CO}$ (ads) interactions [as well as $\mathrm{O}$ (ads)-O(ads) interactions beyond strong $\mathrm{NN}$ repulsions] will of course have a significant effect 
on local ordering within the steady states (cf. Ref. 22), and thus on the reaction kinetics. We do expect that the existence of a nonequilibrium order-disorder transition in the $\mathrm{O}$ (ads) overlayer will be preserved due to the strong short-range $\mathrm{O}$ (ads) $-\mathrm{O}$ (ads) repulsions. However, this transition could be converted to first-order by the presence of $\mathrm{CO}$ (ads) $-\mathrm{O}$ (ads) repulsions, i.e., these interactions could induce "entropic demixing" of $\mathrm{CO}(\mathrm{ads})$ and $\mathrm{O}(\mathrm{ads})$ into separate domains. ${ }^{65}$ Of course, any $\mathrm{CO}(\mathrm{ads})-\mathrm{O}(\mathrm{ads})$ interactions will also directly affect the reaction kinetics. In our modified model without such interactions, reaction is "spatially uniform": $\mathrm{O}(\mathrm{ads})$ interior to $c(2 \times 2)$ domains react at the same rate as those along the domain boundaries [since both have four NN sites which can be populated by $\mathrm{CO}(\mathrm{ads})]$. The presence of $\mathrm{CO}$ (ads) $-\mathrm{O}$ (ads) repulsions of course reduces the population of $\mathrm{CO}$ (ads) on sites interior to $c(2 \times 2)$ domains relative to those on the boundary. This effect would tend to reduce the reactivity of interior $\mathrm{O}$ (ads). However, it is possible that these interactions can also reduce the activation barrier for reaction of interior $\mathrm{O}$ (ads), so as to roughly compensate for the reduced $\mathrm{CO}$ (ads) population. Since the detailed form of the activation barrier is system specific, one cannot draw any general conclusions. Indeed, for some CO-oxidation systems, reaction appears restricted to $\mathrm{O}(\mathrm{ads})$ domain boundaries ${ }^{22}$ while for others it appears more spatially uniform. ${ }^{66}$

Finally, we return to a more detailed discussion of the nature and treatment of chemical diffusion of $\mathrm{CO}$ (ads) in more realistic models. First, it is appropriate to note that in the modified model with common adsorption sites and no interactions [except infinite $\mathrm{NN} \mathrm{O}(\mathrm{ads})-\mathrm{O}$ (ads) repulsions], the nature of CO-diffusion changes qualitatively if one allows hopping of $\mathrm{CO}$ (ads) beyond NN. Even with just NN hops at rate $h$, and diagonal NN hops at rate $h^{\prime}$, say, longrange diffusion no longer vanishes at any percolation transition. ${ }^{67}$ In fact, for $\theta_{\mathrm{O}}=1 / 2$ where one has perfect $c(2$ $\times 2)-\mathrm{O}$ (ads) ordering, it is easy to show that $D_{\mathrm{CO}, \mathrm{CO}}$ $=2 a^{2} h^{\prime}(>0)$, where $a$ is the surface lattice constant. Thus, since longer-range hops will likely occur in real systems, one could argue that the discussions of percolative transport in Sec. V are not so relevant. While at some level this is the case, in practice, it is reasonable to expect that the rate for longer-range hops is well below that for NN hops. Thus, an appropriate view is that the percolative transport picture from Sec. V of $D_{\mathrm{CO} \text {,co }}$ versus $\theta_{\mathrm{O}}$ for just NN hops is basically correct, except that $D_{\mathrm{CO}, \mathrm{CO}}$ no longer completely vanishes at $\theta_{\mathrm{O}}=\theta_{\text {perc }}$ (i.e., the longer-range hops just smooth out the transition).

Second, we should note that the presence of either $\mathrm{CO}$ (ads) $-\mathrm{O}$ (ads) or $\mathrm{CO}($ ads $)-\mathrm{CO}$ (ads) interactions fundamentally complicates the description of chemical diffusion of $\mathrm{CO}$ (ads), as this no longer reduces to a single-particle diffusion problem. In these cases, $D_{\mathrm{CO}, \mathrm{CO}}$ depends on $\theta_{\mathrm{CO}}$ (as well as $\theta_{\mathrm{O}}$ ), and must be determined by general statistical mechanical procedures for analysis of transport coefficients (cf. Ref. 7 and Appendix C).

\section{GENERALIZATIONS AND CONCLUSIONS}

One could consider numerous other surface reactions where the LH mechanisms produce excitable, unstable, etc., behavior, rather than bistable behavior, ${ }^{1,2}$ but where again chemical diffusion in mixed adlayers of two or more reactants controls spatiotemporal behavior. As an example, consider the reactive removal of mixed $\mathrm{NO}+\mathrm{CO}$ adlayers mediated by dissociation of $\mathrm{NO}(\mathrm{ads})$ into $\mathrm{O}(\mathrm{ads})$ and $\mathrm{N}(\mathrm{ads})$, and where the dissociation process typically requires an adjacent empty site. ${ }^{10,68}$ Then, $\mathrm{O}(\mathrm{ads})$ reacts with $\mathrm{CO}(\mathrm{ads})$ to form $\mathrm{CO}_{2}$ (gas), and $\mathrm{N}$ (ads) recombine to form $\mathrm{N}_{2}$ (gas). A completely covered $\mathrm{NO}+\mathrm{CO}$ surface constitutes an unstable state as dissociation of $\mathrm{NO}$ (ads) is inhibited. If an empty patch is created in an otherwise $\mathrm{NO}+\mathrm{CO}$ covered surface, then a Fisher-Kolmogorov-type ${ }^{69}$ wave of reaction will expand from this patch into the covered surface..$^{10}$ In the hydrodynamic limit, the fronts of such waves achieve their selected form much more slowly (as $\sim 1 / t$ ) than trigger waves, ${ }^{10,45}$ and fluctuations can play a more significant role. ${ }^{69} \mathrm{It}$ is instructive to note that for excess $\mathrm{CO}$ (ads) in the $\mathrm{NO}+\mathrm{CO}$ adlayer covering the surface, buildup of $\mathrm{O}(\mathrm{ads})$ is typically small, so all species with significant present on the surface are highly mobile. In this situation, the local states at various points across the reaction front (i.e., points fixed in a reference frame moving with the front) can be regarded as equilibrium states (in a canonical ensemble) with fixed local coverages.

From the above-given example, it is clear that, in general, a precise treatment of the hydrodynamic limit of LG reaction models will require characterization of chemical diffusion in mixed adlayers with several highly mobile adspecies [e.g., $\mathrm{CO}$ (ads), $\mathrm{NO}$ (ads), $\mathrm{N}(\mathrm{ads})]$, often in the presence of coasdorbed relatively immobile adspecies [e.g., $\mathrm{O}(\mathrm{ads})$ ]. For this reason, in Appendix C, we present for the first time, an overview of behavior for the simple classic problem of a mixture of two types of noninteracting adspecies which hop to $\mathrm{NN}$ empty sites with generally distinct rates.

In summary, in this paper, we have presented a strategy for development and analysis of exact RDEs associated with atomistic lattice-gas models for surface reactions in the relevant hydrodynamic limit. We thus provide (and test with simple models for CO-oxidation) one approach for precisely connecting-the-length-scales from a detailed atomistic description of surface reactions to mesoscale pattern formation.

\section{ACKNOWLEDGMENTS}

We acknowledge valuable discussions and collaborations with Professor Ronald Imbihl and his co-workers. These were initiated during a 1991 visit by J.W.E. to the FritzHaber-Institut der MPG, Berlin, which was sponsored by Professor Gerhard Ertl. This work was supported by the Division of Chemical Sciences, DOE-BES. It was performed at Ames Laboratory, which is operated for the USDOE by Iowa State University under Contract No. W-7405-Eng-82.

\section{APPENDIX A: CO-OXIDATION WITH HIGH REACTION RATE}

In the regime of high reaction rate, $k \rightarrow \infty$, a special feature emerges due to both rapid reaction and rapid diffusion of $\mathrm{CO}$ (ads): at each macroscopic spatial point, just one species 
(CO or $\mathrm{O}$ ) can have a nonzero coverage. ${ }^{17,21,30,37}$ Thus, the reactive state has $\theta_{\mathrm{O}}>0$ and $\theta_{\mathrm{CO}}=0$. The chemical wave separating reactive and inactive states has a singular form: diffusion is not relevant in the reactive state, and is trivial in the inactive state [where only noninteracting $\mathrm{CO}$ (ads) is present]. The latter feature facilitates exact analysis of equistability to obtain $P^{*}(\infty)=4 / 7=0.571^{17,37,70}$ (cf. $P_{-}=0$, $\left.P_{+}=2 / 3\right)$ for both standard and modified models with $d$ $=0$.

\section{APPENDIX B: BISTABILITY AND EQUISTABILITY IN SMALL SYSTEMS $\left(L \ll L_{c}\right)$}

It has been claimed that bistability disappears for LG models of CO-oxidation in sufficiently small systems based on explicit calculations showing that the steady state $\theta_{\mathrm{CO}}$ increases monotonically with $P_{\mathrm{CO}}$ (rather than displaying a bistable loop). ${ }^{71}$ However, it follows as a general result from the theory of finite-state Markov processes ${ }^{72}$ that for any finite system (no matter how large), the true steady state is unique, so no bistable loops can ever appear! Instead of considering $\theta_{\mathrm{CO}}$ vs $P_{\mathrm{CO}}$, the appropriate criterion for bistability comes from considering the probability distribution for various populations of adsorbed species. If this distribution is bimodal (with the two peaks corresponding to reactive and inactive "states"), then the system can be characterized as bistable. In fact, we do find bimodal distributions even for very small systems (under suitable conditions of small $d$, etc.). Results will be reported elsewhere. We note that the unique true steady state actually corresponds to the system spending some fraction of time in each of the reactive and inactive "states." (In assessing the true steady state, one must average behavior over sufficiently long times so the system can make very many transitions between these reactive and inactive states.) The fraction of time spent in each state varies smoothly with $P_{\mathrm{CO}}$ explaining the monotonic variation of $\theta_{\mathrm{CO}}$.

One other general issue related to these probability distributions for small systems with $L \ll L_{C}$ is that here one naturally determines equistability of the reactive and inactive "states" as corresponding to equal populations or weights of the two peaks. ${ }^{29}$ How does this criterion compare with equistability determined for the same model from stationarity of chemical waves in a system with $L \gg L_{C}$ ? We claim that the two criteria will in general differ.

Consider the standard model for CO-oxidation with both adspecies highly mobile, i.e., the hop rate for $\mathrm{O}$ (ads), as well as $\mathrm{CO}(\mathrm{ads})$, is large relative to other rates. ${ }^{37}$ (This regime may be unrealistic, but it is instructive.) We know that the equistability point determined from chemical wave analysis in a large system with $L \gg L_{C}$ depends on the detailed specification of adspecies mobility (e.g., different results are obtained for different ratios of hop rates for $\mathrm{CO}$ and O) ${ }^{37} \mathrm{How}-$ ever, in a small system with $L \ll L_{C}$, the adlayer is randomly mixed, so one can write down exact MF master equations for the probability distribution which are independent of the specification of mobility. Thus, the determination of equistability based on the population distribution must also be independent of the detailed specification of mobility.

\section{APPENDIX C: CHEMICAL DIFFUSION IN MIXED ADLAYERS}

Despite the importance of chemical diffusion in mixed adlayers for determining pattern formation in surface reactions, there has been surprisingly little analysis of these problems. Some general discussion is provided in Refs. 47 and 74. However, there is not even available a comprehensive and exact analysis of the simple classic problem of mixed adlayers with two noninteracting adspecies, denoted $\mathrm{A}$ and $\mathrm{B}$, which hop to adjacent empty sites with rates $h_{\mathrm{A}}$ and $h_{\mathrm{B}}$ (per direction), respectively. Here the adlayer is randomly mixed. Reference 47 gives only a simple approximate analysis, and Ref. 74 gives only a partial (exact) analysis, prompting our more complete analysis of this problem. ${ }^{75}$ In the above, A could be $\mathrm{CO}$ (ads), and $\mathrm{B}$ could be $\mathrm{O}$ (ads) (for $\mathrm{CO}$ oxidation) or $\mathrm{NO}$ (ads) (for the $\mathrm{NO}+\mathrm{CO}$ reaction), etc. By symmetry, knowledge of $D_{\mathrm{AA}}$ and $D_{\mathrm{AB}}$ for all $h_{\mathrm{A}} / h_{\mathrm{B}}$ determines the behavior of $D_{\mathrm{BA}}$ and $D_{\mathrm{BB}}$.

From general considerations of transport theory, ${ }^{47,74}$ gradients in the coverages induce diffusive fluxes, $\mathbf{J}_{\mathrm{A}}$ and $\mathbf{J}_{\mathrm{B}}$, for species $\mathrm{A}$ and $\mathrm{B}$, respectively. Arranging coverages and fluxes into two-component vectors, $\boldsymbol{\theta}$ and $\mathbf{J}$, then these are related by ${ }^{8,10,37,47,74}$

$$
\mathbf{J}=-\mathcal{D} \cdot \nabla \boldsymbol{\theta} \text { for small } \nabla \boldsymbol{\theta} .
$$

The $2 \times 2$ diffusion tensor, $\mathcal{D}$, has components, $D_{\alpha \beta}$ with $\alpha$, $\beta=\mathrm{A}$ or $\mathrm{B} . \mathcal{D}$ is not diagonal, i.e., a gradient in the concentration of one species induces a flux in the other. If $h_{\mathrm{B}}=0$, corresponding to the case of CO-oxidation, then $\mathbf{J}_{\mathrm{B}}=\mathbf{0}$ since $D_{\mathrm{BA}}=D_{\mathrm{BB}}=0$. In the following, we list some general properties of the $D_{\alpha \beta}$ in certain extreme coverage regimes: ${ }^{10,37}$

$$
D_{\alpha \beta} \rightarrow D_{\alpha} \delta_{\alpha \beta} \text {, as both } \theta_{\alpha} \rightarrow 0 \text { and } \theta_{\beta} \rightarrow 0 \text { (where }
$$
$\left.D_{\alpha}=a^{2} h_{\alpha}\right)$,

ii) $\quad D_{\mathrm{AA}} \rightarrow D_{\mathrm{tr}}\left(\theta_{\mathrm{B}}\right)$, as $\theta_{\mathrm{A}} \rightarrow 0$, and $D_{\mathrm{BB}} \rightarrow D_{\mathrm{tr}}\left(\theta_{\mathrm{A}}\right)$, as $\theta_{\mathrm{B}}$ $\rightarrow 0$,

(iii) $\quad D_{\mathrm{AB}} \rightarrow 0$, as $\theta_{\mathrm{A}} \rightarrow 0$, and $D_{\mathrm{BA}} \rightarrow 0$, as $\theta_{\mathrm{B}} \rightarrow 0$,

(iv) $D_{\mathrm{AA}}=D_{\mathrm{AB}}$ and $D_{\mathrm{BA}}=D_{\mathrm{BB}}$, when $\theta=\theta_{\mathrm{A}}+\theta_{\mathrm{B}}=1$ (jamming).

Condition (i) reflects independent diffusion of the two adspecies when coverages for both are low. In (ii), generalized tracer diffusion coefficients, $D_{\text {tr }}$, appear corresponding to a single species diffusing through a "bath" of the other species which generally has a different hop rate. ${ }^{56,73}$ Condition (iii) indicates that the flux for species $\alpha$ induced by a gradient in the coverage of the other species must vanish as $\theta_{\alpha} \rightarrow 0$; (iv) follows since diffusion cannot occur when $\theta=1$. It should be noted that the case $h_{\mathrm{A}}=h_{\mathrm{B}}$ is exactly solvable in terms of the tracer diffusion coefficient for a noninteracting single-species lattice gas. $8,10,37,76$

A procedure for comprehensive and exact analysis of such diffusion follows from the statistical mechanical theory for transport processes, where diffusion tensors are calculated in terms of mobility and compressibility tensors. See Refs. 74 and 75 for details. Here, we focus on $D_{\mathrm{AA}} \cdot{ }^{75}$ Figure 10 shows that $D_{\mathrm{AA}}$ naturally decreases with increasing coverage $\theta_{\mathrm{B}}$ of sites "blocking" A diffusion. In general, behavior is described by a family of curves, labeled by $\theta_{\mathrm{A}}$. The 


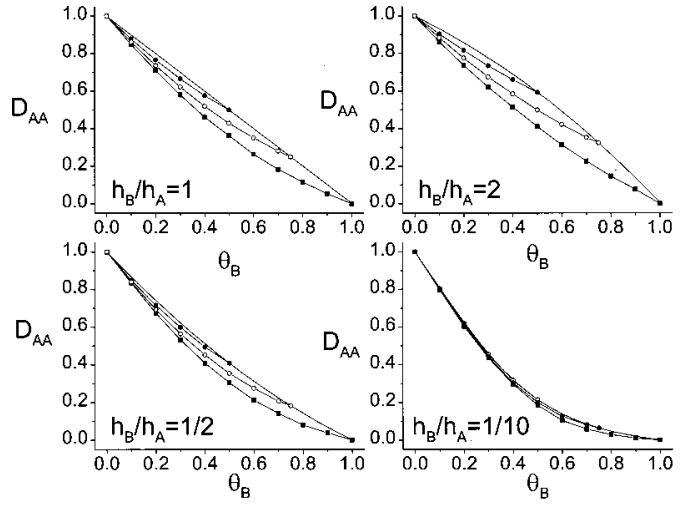

FIG. 10. Results for the diffusion coefficient, $D_{\mathrm{AA}}$, vs $\theta_{\mathrm{B}}$ for a random binary mixture of $\mathrm{A}$ and $\mathrm{B}$ particles on a square lattice, where hopping to NN empty sites occurs at rate $h_{\mathrm{A}}$ for A, and $h_{\mathrm{B}}$ for B (Ref. 75). For each choice of $h_{\mathrm{B}} / h_{\mathrm{A}}, D_{\mathrm{AA}_{3}}$ curves are shown for $\theta_{\mathrm{A}}=\frac{1}{2}, \frac{1}{4}$, and $0+$ (for which $\theta_{\mathrm{B}}$ ranges from 0 to $\frac{1}{2}, \frac{3}{4}$, and 1 , respectively, noting that $\theta_{\mathrm{A}}+\theta_{\mathrm{B}} \leqslant 1$ ). Exact results are available for $h_{\mathrm{B}} / h_{\mathrm{A}}=1$ (Refs. 8, 10, and 76), and behavior for $h_{\mathrm{B}} / h_{\mathrm{A}}=1 / 10$ approaches percolative diffusion of A through coadsorbed immobile B (where $D_{\mathrm{AA}}$ is independent of $\theta_{\mathrm{A}}$ ).

lower envelope of the family corresponds to $D_{\mathrm{AA}}$ $\rightarrow D_{\text {tr }}\left(\theta_{\mathrm{B}}\right)$, for $\theta_{\mathrm{A}} \rightarrow 0$. The upper envelope of the family of curves corresponds to the jammed limit where $\theta_{\mathrm{B}}=1-\theta_{\mathrm{A}}$. The one case when the family of $D_{\mathrm{AA}}$ curves collapses to a single curve is for "percolative diffusion" when $h_{\mathrm{B}} / h_{\mathrm{A}} \rightarrow 0$ (cf. Sec. IV). Note that for fixed coverages, $D_{\mathrm{AA}}$ decreases with decreasing $h_{\mathrm{B}} / h_{\mathrm{A}}$, i.e., the less mobile the coadsorbed species, the greater the inhibition of diffusion (so quenched disorder has more impact than dynamic disorder). Finally, we remark that $D_{\mathrm{AB}}$ naturally increases with increasing coverage $\theta_{\mathrm{A}}$ : the diffusive flux of $\mathrm{A}$ induced by a gradient $\nabla \theta_{\mathrm{B}}$ should increase with $\theta_{\mathrm{A}} \cdot{ }^{75}$

${ }^{1}$ G. Ertl, Adv. Catal. 37, 213 (1991); R. Imbihl, Prog. Surf. Sci. 44, 185 (1993).

${ }^{2}$ M. Eiswirth and G. Ertl, in Chemical Waves and Patterns, edited by R. Kapral and K. Showalter (Kluwer, Dordrecht, 1994); R. Imbihl and G. Ertl, Chem. Rev. 95, 697 (1995).

${ }^{3}$ T. Engel and G. Ertl, Adv. Catal. 28, 1 (1979).

${ }^{4}$ L. F. Razon and R. A. Schmitz, Catal. Rev. Sci. Eng. 28, 89 (1986).

${ }^{5}$ M. Berdau, G. G. Yelenin, A. Karpowicz, M. Ehsasi, K. Christmann, and J. H. Block, J. Chem. Phys. 110, 11551 (1999).

${ }^{6}$ N. Gottschalk, F. Mertens, M. Eiswirth, and R. Imbihl, Phys. Rev. Lett. 73, 3483 (1994) is a rare example utilizing nonconstant diffusion coefficients to describe the influence of surface reconstruction.

${ }^{7}$ R. Gomer, Rep. Prog. Phys. 53, 917 (1990).

${ }^{8} \mathrm{H}$. Spohn, Large Scale Dynamics in Interacting Particle Systems (Springer, Berlin, 1991).

${ }^{9}$ M. Tammaro and J. W. Evans, J. Chem. Phys. 108, 762 (1998).

${ }^{10}$ M. Tammaro and J. W. Evans, J. Chem. Phys. 108, 7795 (1998).

${ }^{11}$ D.-J. Liu and J. W. Evans, J. Chem. Phys. 113, 10252 (2000).

${ }^{12}$ W. Swiech, B. Rausenberger, R. Imbihl, J. W. Evans, C. S. Rastomjee, W. Engel, A. K. Schmid, A. M. Bradshaw, and E. Zeitler, Surf. Sci. 307-309, 138 (1994)

${ }^{13}$ A. S. Mikhailov and G. Ertl, Chem. Phys. Lett. 238, 104 (1995); V. P. Zhdanov, ibid. 267, 397 (1997).

${ }^{14}$ M. Hildebrand and A. S. Mikhailov, J. Phys. Chem. 100, 19089 (1996); M. Hildebrand, A. S. Mikhailov, and G. Ertl, Phys. Rev. Lett. 81, 2602 (1998); M. Hildebrand and A. S. Mikhailov, J. Stat. Phys. 101, 599 (2000).

${ }^{15}$ W. H. Weinberg, Annu. Rev. Phys. Chem. 34, 217 (1983); K. Binder and D. P. Landau, Adv. Chem. Phys. 26, 91 (1989).

${ }^{16}$ R. M. Ziff, E. Gulari, and Y. Barshad, Phys. Rev. Lett. 56, 2553 (1986).

${ }^{17}$ J. W. Evans, J. Chem. Phys. 97, 572 (1992); 98, 2463 (1993).

${ }^{18}$ J. W. Evans and T. R. Ray, Phys. Rev. E 50, 4302 (1994).
${ }^{19}$ R. H. Goodman, D. S. Graff, L. M. Sander, P. Leroux-Hugon, and E. Clement, Phys. Rev. E 52, 5904 (1995); E. V. Albano, ibid. 55, 7144 (1997).

${ }^{20}$ H. C. Kang, T. A. Jachimowiski, and W. H. Weinberg, J. Chem. Phys. 93, 1418 (1990); K. A. Fichthorn and W. H. Weinberg, ibid. 95, 1090 (1991).

${ }^{21}$ V. P. Zhdanov and B. Kasemo, Surf. Sci. 412/413, 527 (1998).

${ }^{22}$ S. Volkening and J. Wintterlin, J. Chem. Phys. 114, 6382 (2001).

${ }^{23}$ M. Silverberg and A. Ben-Shaul, J. Chem. Phys. 87, 3178 (1989); Chem. Phys. Lett. 134, 491 (1987); J. Chem. Phys. 87, 3178 (1987); M. Silverberg, A. Ben-Shaul, and F. Rebentrost, ibid. 83, 6501 (1985).

${ }^{24}$ M. Tammaro, M. Sabella, and J. W. Evans, J. Chem. Phys. 103, 10277 (1995).

${ }^{25}$ E. W. James, C. Song, and J. W. Evans, J. Chem. Phys. 111, 6579 (1999).

${ }^{26}$ A. P. J. Jansen and J. J. Lukkien, Catal. Today 53, 259 (1999).

${ }^{27}$ More generally, one could include hops to nearest-neighbor (NN) sites with rate $h$, and to next NN sites with rate $h^{\prime}$ (likely much smaller than $h$ ), etc.

${ }^{28}$ M. Dumont, P. Dufour, B. Sente, and R. Daggonier, J. Catal. 122, 95 (1990).

${ }^{29}$ Y. Suchorski, J. Beben, E. W. James, J. W. Evans, and R. Imbihl, Phys. Rev. Lett. 82, 1907 (1999); Y. Suchorski, J. Beben, R. Imbihl, E. W. James, D.-J. Liu, and J. W. Evans, Phys. Rev. B 63, 165417 (2001).

${ }^{30}$ D.-J. Liu and J. W. Evans, Phys. Rev. Lett. 84, 955 (2000).

${ }^{31}$ C. R. Brundle, R. J. Behm, and J. A. Barker, J. Vac. Sci. Technol. A 2, 1038 (1984); S.-L. Chang and P. A. Thiel, Phys. Rev. Lett. 59, 296 (1987).

${ }^{32}$ For $d=0$, the steady-state or bifurcation diagram is somewhat anomalous, displaying a transcritical bifurcation at $P_{\mathrm{CO}}=0$, but this does not affect the issues of interest here. In fact, the existence of this "absorbing" state, in the language of Markov processes, actually facilitates precise analysis of poisoning transitions.

${ }^{33}$ A. S. Mikhailov, Foundations of Synergetics I (Springer, Berlin, 1990).

${ }^{34}$ D.-J. Liu and J. W. Evans (unpublished). For our modified model with $d$ $=0$ and $k=1$, where the CO-poisoning transition changes from discontinuous to continuous, for small $h$.

${ }^{35}$ J. W. Evans and M. S. Miesch, Phys. Rev. Lett. 66, 833 (1991).

${ }^{36}$ Extremely weak metastability appears in the standard model (for finite $k$, or in the ZGB model with $k=\infty$ ) when $h=0$. See Refs. 18 and 35 and R. A. Monetti and E. V. Albano, J. Phys. A 34, 1103 (2001).

${ }^{37}$ J. W. Evans and M. Tammaro, in Computer Simulation Studies in Condensed Matter Physics XI, edited by D. P. Landau and H.-B. Schuettler (Springer, Berlin, 1999), p. 103; Phys. Rev. E 57, 5087 (1998).

${ }^{38}$ One can show that a discrete version of MF RDEs, with "simple" diffusive coupling described by a discretized Laplacian, produces a weaker shift of the form $\delta P^{*}(h) \sim 1 / h$, as $h \rightarrow \infty$.

${ }^{39} \mathrm{~A}$ precise analysis is possible for $d=0$ when the metastable state is "absorbing." Then, one can unambiguously follow the fate of the embedded blob (whereas for $d>0$, a blob could disappear and spontaneously reappear). Also, for $d=0$, perpetual propagation of stable reactive state into inactive state occurs for $P_{\mathrm{CO}}<P^{*}$ (whereas the inactive state eventually breaks down for $d>0$ ).

${ }^{40} \mathrm{~A}$ heuristic treatment of the relationship between $R_{c}$ and the lifetime can be found in V. P. Zhdanov, Phys. Rev. E 50, 760 (1994). See also E. V. Albano and J. Marro, J. Chem. Phys. 113, 10279 (2000).

${ }^{41}$ J. W. Evans, Langmuir 7, 2514 (1991).

${ }^{42}$ B. Brosilow and R. M. Ziff, Phys. Rev. A 46, 4534 (1992).

${ }^{43}$ T. Tome and R. Dickman, Phys. Rev. E 47, 948 (1993).

${ }^{44}$ J. W. Evans and M. Sabella, Trends Stat. Phys. 1, 107 (1994).

${ }^{45}$ U. Ebert and W. van Saarloos, Phys. Rev. Lett. 80, 1650 (1998).

${ }^{46}$ Significant values of $\xi$ relative to $R_{c}$ produce substantial smearing of $\mathrm{P}(R)$-Ref. 18. KPZ analysis also shows that $\xi_{0}$ is small on a length scale $L_{c}$, for large $h$ (Ref. 9).

${ }^{47}$ V. P. Zhdanov, Surf. Sci. 194, 1 (1988).

${ }^{48}$ R. M. Ziff and B. Brosilow, Phys. Rev. A 46, 4560 (1992).

${ }^{49}$ F. H. Ree and D. A. Chesnut, J. Chem. Phys. 45, 3983 (1966).

${ }^{50}$ D.-J. Liu and J. W. Evans, Phys. Rev. B 62, 2134 (2000).

${ }^{51}$ E. W. James, D.-J. Liu, and J. W. Evans (unpublished).

${ }^{52}$ One has $S_{\mathrm{O}_{2}}=\left[\theta_{E} /\left(1-\theta_{\mathrm{O}}\right)\right]^{2} S_{8}$, where $S_{8} \approx\left[\left(1-2 \theta_{\mathrm{O}}\right) /\left(1-\theta_{\mathrm{O}}\right)\right]^{8}$ is the pair approximation for the probability of an adsorption ensemble of eight sites not occupied by $\mathrm{O}$ (ads) - Ref. 25.

${ }^{53}$ W. E. Schiesser, The Numerical Methods of Lines (Academic, San Diego, 1991). 
${ }^{54}$ Compare $P^{*}(\infty)=0.397$ with $P_{-}(\infty)=0, P_{\mathrm{O}_{\text {poison }}}=0.378$, and $P_{+}(\infty)$ $=0.500$ (standard model).

${ }^{55}$ Compare $P^{*}(\infty)=0.325$ with $P_{-}(\infty)=0$ and $P_{+}(\infty)=0.360$ (modified model).

${ }^{56}$ R. Kutner and K. W. Kehr, Philos. Mag. A 48, 199 (1983).

${ }^{57}$ A. Bunde and S. Havlin, in Fractals and Disordered Systems, edited by A. Bunde and S. Havlin (Springer, Berlin, 1991), Chaps. 2 and 3.

${ }^{58}$ P. G. de Gennes, Recherche 7, 919 (1976).

${ }^{59}$ T. M. Nieuwenhuizen, P. F. J. van Velthoven, and M. H. Ernst, Phys. Rev. Lett. 57, 2477 (1986).

${ }^{60}$ One can write down a rate equation for the population of "monomers" which are created by reaction of one of the constituent B's of a just deposited "dimer," and destroyed by reaction. For the standard model, the analysis in Ref. 9 incorrectly set $a_{1}=\pi-1$, and $a_{1}$ reported in Ref. 11 is also incorrect.

${ }^{61}$ G. A. Somorjai, Introduction to Surface Chemistry and Catalysis (Wiley, New York, 1994).

${ }^{62}$ J. Wintterlin, Adv. Catal. 45, 131 (2000).

${ }^{63}$ E. M. Stuve, R. J. Madix, and C. R. Brundle, Surf. Sci. 146, 155 (1984).
${ }^{64}$ B. N. J. Persson, Surf. Sci. Rep. 15, 1 (1992).

${ }^{65}$ D.-J. Liu and J. W. Evans, J. Chem. Phys. 114, 10977 (2001).

${ }^{66}$ M. Xu, J. Liu, and F. Zaera, J. Chem. Phys. 104, 8825 (1996).

${ }^{67}$ See Appendix F of Ref. 11.

${ }^{68}$ Th. Fink, J.-P. Dath, M. R. Bassett, R. Imbihl, and G. Ertl, Surf. Sci. 245, 96 (1991).

${ }^{69}$ J. Riordan, C. R. Doering, and D. ben-Avraham, Phys. Rev. Lett. 75, 565 (1995); J. Mai, I. M. Sokolov, and A. Blumen, ibid. 77, 4462 (1996); D. A. Kessler, Z. Ner, and L. M. Sander, Phys. Rev. E 58, 107 (1998).

${ }^{70}$ V. P. Zhdanov and B. Kasemo, Surf. Sci. Rep. 20, 111 (1994).

${ }^{71}$ A. V. Zhdanova, Phys. Rev. B 63, 153410 (2001).

${ }^{72}$ N. G. Van Kampen, Stochastic Processes in Physics and Chemistry (North-Holland, Amsterdam, 1981).

${ }^{73}$ R. A. Tahir-Kheli and R. J. Elliot, Phys. Rev. B 27, 844 (1983).

${ }^{74}$ K. W. Kehr, K. Binder, and S. M. Reulin, Phys. Rev. B 39, 4891 (1989).

${ }^{75}$ M. Tammaro and J. W. Evans (unpublished) corrects the form proposed in M. Tammaro and J. W. Evans, Surf. Sci. Lett. 395, L207 (1998) for $D_{\mathrm{AA}}$ when $h_{\mathrm{B}} / h_{\mathrm{A}}>1$.

${ }^{76}$ J. Quastel, Commun. Pure Appl. Math. 45, 623 (1992). 